\title{
Chicago sky blue 6B (CSB6B), an allosteric inhibitor of macrophage migration inhibitory factor (MIF), suppresses osteoclastogenesis and promotes osteogenesis through the inhibition of the NF-KB signaling pathway.
}

\section{Kangtao Jin}

Lishui Central Hospital and Fifth Affiliated Hospital of Wenzhou Medical College

\section{Lin Zheng}

Lishui Central Hospital and Fifth Affiliated Hospital of Wenzhou Medical College

\section{Ziang Xie}

Zhejiang University School of Medicine Sir Run Run Shaw Hospital

\section{Lin Ye}

Lishui Central Hospital and Fifth Affiliated Hospital of Wenzhou Medical College Jiawei Gao

Lishui Central Hospital and Fifth Affiliated Hospital of Wenzhou Medical College Chao Lou

Lishui Central Hospital and Fifth Affiliated Hospital of Wenzhou Medical College Wenzheng Pan

Lishui Central Hospital and Fifth Affiliated Hospital of Wenzhou Medical College Bin Pan

Lishui Central Hospital and Fifth Affiliated Hospital of Wenzhou Medical College Shijie Liu

Lishui Central Hospital and Fifth Affiliated Hospital of Wenzhou Medical College

\section{Zhenzhong Chen}

Lishui Central Hospital and Fifth Affiliated Hospital of Wenzhou Medical College

Dengwei He ( $\nabla$ hedw_spine@163.com )

Lishui Central Hospital and Fifth Affiliated Hospital of Wenzhou Medical College https://orcid.org/0000-0003-2394-2124

Research

Keywords: osteoclast, osteoblast, osteoporosis, MIF, CSB6B 
Posted Date: May 19th, 2020

DOI: https://doi.org/10.21203/rs.3.rs-26854/v1

License: (9) This work is licensed under a Creative Commons Attribution 4.0 International License. Read Full License

Version of Record: A version of this preprint was published at Biochemical Pharmacology on August 1st, 2021. See the published version at https://doi.org/10.1016/j.bcp.2021.114734. 
1 Chicago sky blue 6B (CSB6B), an allosteric inhibitor of macrophage migration

through the inhibition of the NF-кB signaling pathway.

Kangtao Jin ${ }^{1}$, Lin Zheng ${ }^{1,2,3, \dagger}$, Ziang Xie ${ }^{2,3}$, Lin Ye ${ }^{1}$, Jiawei Gao ${ }^{1}$, Chao Lou ${ }^{1}$, Wenzheng Pan $^{1}$, Bin Pan $^{1}$, Shijie Liu ${ }^{1}$, Zhenzhong Chen ${ }^{1,3}$ and Dengwei He ${ }^{1, *}$

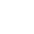

${ }^{1}$ Department of Orthopedics, Affiliated Lishui Hospital of Zhejiang University/the Fifth Affiliated Hospital of Wenzhou Medical University/Lishui Central Hospital, Lishui, China.

${ }^{2}$ Department of Orthopaedic Surgery, Sir Run Run Shaw Hospital, Zhejiang University School of Medicine, Hangzhou, China.

${ }^{3}$ Key Laboratory of Musculoskeletal System Degeneration and Regeneration Translational Research of Zhejiang Province, Hangzhou, China.

$\dagger$ These authors contributed equally to this work.

*Correspondence: Department of Orthopedics, Affiliated Lishui Hospital of Zhejiang University, the Fifth Affiliated Hospital of Wenzhou Medical University, Lishui Central Hospital, 289 Kuocang Rd., Lishui 323000, China. E-mail: hedw_spine@163.com

Kangtao Jin: jkttt1994@163.com, Lin Zheng: wzz192@163.com , Ziang Xie: zia ng_xie@zju.edu.cn, Lin Ye: 407404159@qq.com, Jiawei Gao: 21818368@zju.ed u.cn, Chao Lou: 564776324@qq.com, Wenzheng Pan: panwenzheng1996@163.c om, Bin Pan: 1621583798@qq.com, Shijie Liu: imliushijie@126.com, Zhenzhon g Chen: 379095641@qq.com, and Dengwei He: hedw_spine@163.com. 
ABSTRACT

Background : Macrophage migration inhibitory factor (MIF) is a pleiotropic proinflammatory mediator involved in various pathophysiological and inflammatory states.Accumulating line of evidence suggests a role for MIF in regulating bone metabolism and therefore a prime candidate for therapeutic targeting. In this study, we showed that Chicago sky blue 6B (CSB6B), an unique allosteric inhibitor of MIF catalytic and cytokine activity, suppresses RANKL-induced osteoclast and promotes osteogenesis in vitro via the inhibition of NF- $\kappa \mathrm{B}$ signaling activation

Methods: We examined the effects of CSB6B on osteoclast differentiation and bone resorption and the bone formation ability of osteoblasts in vitro. The effect of CSB6B on the NF- $\kappa$ B pathway was subsequently detected using western blotting and Co-IP. Finally, the model of mouse skull dissolution and ovarian severing were modeled and intraperitoneally injected with different doses of CSB6B to observe the anti-osteolytic and anti-osteoporosis effects of the drug in vivo.

Results: In this study, we showed that Chicago sky blue 6B (CSB6B) suppresses RANKL-induced osteoclast and bone resorption in vitro via the inhibition of NF- $\mathrm{KB}$ signaling activation and promoting proteasome-mediated degradation of MIF. Consequently, the induction of NFATc1 was impaired resulting in downregulation of NFATc1-responsive osteoclast genes. We also demonstrated that CSB6B treatment enhanced primary calvarial osteoblast differentiation and bone mineralization in vitro via the suppression of NF- $\mathrm{BB}$ activation and upregulation of Runx expression. Using two murine models of osteolytic bone disorders, we further showed that administration of CSB6B protected mice against pathological inflammatoryc calvarial bone destruction induced by titanium particles mice as well as estrogen-deficiency induced bone loss as a result of ovariectomy.

Conclusion: Together, as an MIF inhibitor, CSB6B can inhibit osteoclast differentiation and absorption function and enhance the mineralization of osteoblasts through the inhibition of NF- $\mathrm{kB}$ pathway. MIF is a prime target for therapeutic targeting for the treatment of osteolytic bone disorders and the MIF inhibitor CSB6B could be 
potential anti-osteoporosis drug.

KEY WORDS: osteoclast, osteoblast, osteoporosis, MIF, CSB6B

\section{Background}

Bone remodeling is a dynamic process balanced by osteoclast-mediated bone resorption and osteoblast-mediated bone formation [1,2]. Imbalances in osteoclast and osteoblast activities favouring excessive osteoclast formation and/or bone resorption leads to vairous metabolic bone disease including estrogen-deficiency mediated bone loss as in post-menopausal osteoporosis, and inflammatory bone destruction associated with pathological osteolysis[3]. Although some level of effectiveness against osteoclastmediated bone loss is achieved with currently available anti-resorptive agents, side effects including gastrointestinal, renal and ocular toxicities, osteonecrosis of the jaw, and atypical fractures have been reported [4]. Thus, there is an urgent need for newer, safer and more effective treatment options for the treatment of osteolytic diseases. Macrophage migration inhibitory factor (MIF), is a pleiotropic pro-inflammatory mediator that has important functions in the regulation of the innate and adaptive immune response[5-7]. MIF is expressed by numerous immune cells as well as by cells beyond the immune system such as epithelial, endocrine, smooth muscle, and endothelial cells[7]. As such MIF exerts a wide variety of biological functions and involved in variety of pathophysiological and inflammatory states. In fact, there mounting evidence for a role of MIF in the development of various osteolytic bone conditions[8-11]. MIF deficient mice have been shown to be protected against OVXinduced bone loss whereas transgenic mice overexpressing MIF exhibit elevated 
osteoclastic bone resorption[11] and high bone turnover osteoporosis[12]. In a recent paper, previous showed that a suicide substrate that binds MIF and inhibits its activity suppresses osteoclast formation[13]. Thus MIF is a prime target for the development or identification of therapeutic agents for the treatment of osteolytic bone conditions. Chicago sky blue 6B (CSB6B), is an azo compound identified through high throughput screening that potently inhibits MIF tautomerase activity[14]. It is a uniquely rare allosteric inhibitor that binds at the interface of two MIF timers blocking the catalytic and cytokine activities of MIF [14]. Here in this study we investigated the effects of CSB6B on bone metabolism, in the context of RANKL-induced osteoclast formation and bone resorption, and osteoblast differentiation and bone mineralization in vitro. We also examined the potential in vivo therapeutic effects of CSB6B administration in pathological inflammatory calvarial bone destruction induced by titanium particles and estrogen-deficiency bone loss induced by ovariectomy.

\section{Materials and Methods}

\section{Experimental animals}

All animal experiments conducted in this study were approved by the Ethics Committee of Sir Run Run Shaw Hospital (Zhejiang University, Zhejiang, China) and carried out in accordance with the institute's guidelines for the treatment of animals and to the principles from the National Institute of Health's Guide for the Care and Use of Laboratory Animals (NIH; Bethesda, MD, USA). Eight-week-old C57BL/6J male MIF knockout (KO) and wild-type (WT) littermates were used in downstream experiments. MIF KO mice was generated via the inactivation of the $\underline{M I F}$ gene through the substitution of part of exon 2, the second intron, and exon 3 with the neomycin gene cassette. Tail-tip genotyping of KO mice were carried out using semi-quantitative PCR analysis with the following primer sets: Primer 1 (Forward: 5'CCAACCTACAGGTTCCACCAATAAC-3', and 5'GCCCAGCTGGAGCACACTATT-3'); and Primer 2 (Forward: 5'TAGACCACGTGCTTAGCTGAGCC-3', and 5'GCCCAGCTGGAGCACACTATT-3'). 


\section{Media and Reagents}

Dulbecco's modified Eagle Medium (DMEM), alpha modification of Eagle's minimal essential medium ( $\alpha$-MEM), fetal bovine serum (FBS), and penicillin-streptomycin were purchased from Thermo Fisher Scientific (Waltham, MA, USA). Chicago Sky Blue 6B (CSB6B) was obtained from Tocris Bioscience (Bristol, UK) and dissolved in PBS to stock concentration of $100 \mathrm{mM}$ and stored at $-80^{\circ} \mathrm{C}$. CSB6B is further diluted to working concentration in culture media when needed. Recombinant mouse macrophage-colony stimulating factor (M-CSF) and receptor activator of nuclear factor- $\kappa \mathrm{B}$ ligand (RANKL) were procured from R\&D Systems (Minneapolis, MN, USA). Primary antibodies against mouse NF- $\kappa$ B subunit p65, phospho-p65 (Ser536),

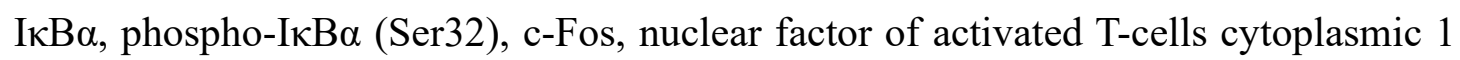
(NFATc1), runt-related transcription factor 2 (Runx2) and $\beta$-actin were from Cell Signaling Technology (Danvers, MA, USA). Primary antibodies against MIF were purchased from Abcam (Cambridge, UK). TRAP staining kit was from MilliporeSigma (Burlington, MA, USA).

\section{Primary bone marrow monocytes/macrophage (BMMs) isolation}

Primary mouse bone marrow monocytes/macrophages (BMMs) were extracted from the long bones of mice by marrow flushing as previously described by Zheng et al[13]. Extracted BMMs were cultured in $\alpha$-MEM containing $10 \%$ FBS and $1 \%$ penicillinstreptomycin (complete $\alpha-\mathrm{MEM}$ ) supplemented with $40 \mathrm{ng} / \mathrm{ml} \mathrm{M-CSF}$ and maintained in humidified $\left(95 \%\right.$ air $\left./ 5 \% \mathrm{CO}_{2}\right)$ incubator at $37^{\circ} \mathrm{C}$ until the cell monolayer reached $90 \%$ confluence.

\section{Cytotoxicity assay}

The cellular cytotoxicity of CSB6B were examined using the Cell Counting Kit-8 (CCK-8; Dojindo Laboratories, Kumamoto, Japan) assay as per manufacturer's protocol. Cells seeded into 96-well plates at a density of $2 \times 10^{4}$ cells/well were treated with various concentrations of CSB6B $(0.75,1.5,3.125,6.25,12.5,25,50,100$, and $200 \mu \mathrm{M})$. After 48 or 96 hours incubation with CSB6B, $10 \mu \mathrm{l}$ of CCK-8 reagent was added to each well and incubated for further 2 hours. The absorbance at $450 \mathrm{~nm}$ for each experimental condition were obtained on an ELx800 Absorbance Microplate 
Reader (BioTek Instruments, Winooski, VT, USA).

\section{In vitro osteoclast differentiation and formation assay}

For osteoclast differentiation assay, BMMs were seeded onto 24-well plates at a density of $5 \times 10^{4}$ cells/well in complete $\alpha$-MEM supplemented with $40 \mathrm{ng} / \mathrm{ml} \mathrm{M-CSF}$ for 24 hours. The next day cells were stimulated with $50 \mathrm{ng} / \mathrm{ml}$ RANKL without or with CSB6B at concentrations of 5, 10, and $20 \mathrm{mM}$. Mock control cells were treated with PBS instead of CSB6B. Culture media were changed every other day for a total of 5 7 days or until mature multinucleated osteoclasts were observed in mock controls. Cells were gently and briefly washed with PBS to remove floating cells, fixed with $4 \%$ paraformaldehyde (PFA) for 15 mins, and then stained for TRAP activity. The number of $\mathrm{TRAP}^{+}$osteoclasts with 3 or more nuclei and the average area occupied by the osteoclasts (\% of well) were quantified using ImageJ software (NIH).

\section{In vitro bone resorption assay}

BMMs were seeded on collagen coated plates with RANKL for 3-4 days. Then the preosteoclasts were reseeded onto bone discs in complete media containing MCSF and RANKL with different concentration of CSB6B for extra 3-4 days after cells were settled for 6 hours .At the end of the experiment, cells were removed by gentle brushing and mechanical sonication. Resorption pits were visualized and captured using an FEI Quanta 250 scanning electron microscope (Thermo Fisher Scientific). Bone resorption area was measured using Image $(\mathrm{NIH})$ software and expressed relative to untreated controls.

\section{Podosomal actin belt immunofluorescence}

BMM-derived osteoclasts were generated and treated with CSB6B as described in osteoclast differentiation and formation assay above. When mature multinucleated osteoclasts were observed in RANKL-only treated controls, cells were fixed with 4\% PFA for 15 mins and then permeabilized with $0.1 \%(v / v)$ Triton $\mathrm{X}-100$ for 5 mins at room temperature. Cells were then incubated with rhodamine-conjugated phalloidin (Thermo Fisher Scientific) diluted in $0.2 \%(\mathrm{w} / \mathrm{v})$ bovine serum albumin-PBS for 1 hour at room temperature in the dark. DAPI (Santa Cruz Biotechnology, Dallas, TX, USA) was used to stain the cell nuclei. Fluorescence images were captured using the Nikon 
A1 confocal laser microscope and associated software (Nikon, Tokyo, Japan). ImageJ was used to quantify the number of podosomal actin belts.

\section{In vitro osteoblastogenesis assay}

The effects of CSB6B on osteoblasts were established using primary calvarial osteoblasts extracted from 2-day-old C57BL/6J mice as previously described by Zheng et al (46). Cells were maintained in DMEM containing 10\% FBS and 1\% penicillinstreptomycin (complete DMEM) until 90\% confluence. For osteoblast differentiation and alkaline phosphatase (ALP) activity, calvarial osteoblasts seeded in 24-well plates at density of $5 \times 10^{4}$ cells/well were stimulated with osteogenic media (10 nM dexamethasone, $10 \mathrm{mM} \beta$-glycerophosphate, and $50 \mu \mathrm{g} / \mathrm{ml}$ ascorbic acid) without or with 5,10 , or $20 \mathrm{mM}$ of CSB6B. Osteogenic media was replaced every other day and after 7 days, cells were fixed and stained for ALP activity using BCIP/NBT staining kit (CoWin Biosciences, Beijing, China) according to manufacturer's instructions. For mineralization assays, cells were cultured in osteogenic media without or with 5,10 , or $20 \mathrm{mM}$ of CSB6B for 21 days. Cells were then fixed in 4\% PFA and mineral deposition assessed by staining with Alizarin Red S (ARS) solution (Cyagen Biosciences, Santa Clara, CA, USA) as per manufacturer's protocol. Phase contrast images of ALP and ARS stained cells were acquired on a Zeiss Primovert inverted light microscope (Carl Zeiss AG, Oberkochen, Germany). Quantitative measurements of calcium deposition in the bone nodules were conducted following incubation of ARS-stained nodules with $100 \mathrm{mM}$ cetylpyridinium chloride (CPC) for 1 hour. The absorbance/optical density were measured at wavelength of $550 \mathrm{~nm}$ on an ELx800 Absorbance Microplate Reader (BioTek Instruments).

\section{RNA extraction and real-time quantitative PCR}

Total RNA were extracted from cultured cells using the RNeasy Mini Kit (QIAGEN, Hilden, Germany) in accordance with manufacturer's protocol. Complementary DNAs (cDNAs) were synthesized from $1 \mu \mathrm{g}$ of total RNA using the Prime Script RT Master Mix (Takara Bio, Kusatsu, Japan) based on the guidelines from the manufacturer. The resulting cDNA was used as template together with specific primers in the SYBR Green qPCR Master Mix reaction (Takara Bio). Real-time qPCR was performed on an ABI 
Prism 7500 qPCR system (Applied Biosystems, Thermo Fisher Scientific) with the following reaction parameters: initial denaturation at $95^{\circ} \mathrm{C}$ for $10 \mathrm{mins}$; followed by 40 repeated cycles of $95^{\circ} \mathrm{C}$ for $10 \mathrm{secs}, 60^{\circ} \mathrm{C}$ for $20 \mathrm{secs}$, and $72^{\circ} \mathrm{C}$ for $20 \mathrm{secs}$; with a final extension at $72^{\circ} \mathrm{C}$ for 90 secs. The primer sets targeting the following mouse genes were used: GAPDH (Forward: 5'-GCAAGTTCAACGGCACAG-3', and Reverse: 5'CGCCAGTAGACTCCACGAC-3'); NFATc1

(Forward:

$5^{\prime}-$ GGGTCAGTGTGACCGAAGAT-3', and Reverse: $5^{\prime}-$ GgaAgtCAgaAgtgGgtgGA-3'); Cathepsin $K$ (CTSK) (Forward: 5'TCCGCAATCCTTACCGAATA-3', and Reverse: 5'AACTTGAACACCCACATCCTG-3'); TRAP (Forward: 5'CCATTGTTAGCCACATACGG-3', and Reverse: $\quad 5$ '-

CACTCAGCACATAGCCCACA-3');

GTTCGTGAAACACACCAGGC-3', $c-F o s$

(Forward: 5 GGCCTTGACTCACATGCTCT-3'); T-cell immune regulator 1(TCIRG1) (Forward: 5'-TGGCTACCGTTCCTATCCTG-3', and Reverse: 5 CTTGTCCGTGTCCTCATCCT-3'); dendritic cell-specific transmembrane protein (DC-STAMP) (Forward: 5'-GCTGTATCGGCTCATCTCCT-3', and Reverse: 5'AAGGCAGAATCATGGACGAC-3'); Runx2 (Forward: 5'GCGCATTCCTCATCCCAGTA-3', and ${ }^{\prime}$ TGGAGTGGAtGGATGGGGAT-3'); $\quad$ (Forward: 5'TTCATAAGCAGGCGGGGGA-3', and Reverse: 5 GGTGTACCCTGAGATTCGTCC-3'); $(\mathrm{OCN}) \quad$ (Forward: 5'CCCTGAGTCTGACAAAGCCT-3', and Reverse: 5 GCGGTCTTCAAGCCATACTG-3'); and Osteopontin (OPN) (Forward: 5'TGGAGAGGTAGAAAAGGCACA-3', and Reverse: $5{ }^{\prime}-$ CAAACACACTCTTGGCACCAC-3'). Gene expressions were normalized to the expression of GAPDH using $2^{-\triangle \Delta \mathrm{CT}}$ method. The mean $\mathrm{CT}$ value of target genes in the experimental group was first normalized to the $\mathrm{CT}$ value of $G A P D H$ to give a $\triangle \mathrm{CT}$ value. The $\Delta \mathrm{CT}$ value was then further normalized to control samples to obtain $\Delta \Delta \mathrm{CT}$ value. The expression of target genes were then expressed relative to untreated controls. 


\section{Western blot analysis and co-immunoprecipitation (Co-IP)}

RIPA lysis buffer (MilliporeSigma) containing protease and phosphatase inhibitors (Thermo Fisher Scientific) were used isolate total cell proteins from cultured cells. Cell lysates were cleared by centrifugation and the resulting post-nuclear supernatants were subjected to protein concentration quantification. A total of $20 \mu \mathrm{g}$ of protein sample were resolved on $10 \%$ SDS-PAGE and separated proteins were electroblotted onto PVDF membranes (Bio-Rad Laboratories, Hercules, CA, USA) overnight at $4{ }^{\circ} \mathrm{C}$. Membranes were blocked with 5\% (w/v) skim-milk in Tris buffered saline with Tween 20 [TBST; $150 \mathrm{mM} \mathrm{NaCl}, 50 \mathrm{mM}$ Tris (pH 7.6), 0.1\% Tween-20] for 1 hour at room temperature and then incubated with primary antibodies diluted $1 / 1000$ in $1 \%(\mathrm{w} / \mathrm{v})$ skim-milk in TBST at $4^{\circ} \mathrm{C}$ overnight with gentle agitation. After extensive washes with TBST, membranes were incubated with appropriate horseradish peroxidase-conjugated secondary antibodies diluted $1 / 5000$ in $1 \%(\mathrm{w} / \mathrm{v})$ skim-milk in TBST for 1 hour at room temperature. Protein bands were visualized following incubation with Luminata ${ }^{\mathrm{TM}}$ Western Chemiluminescent HRP substrate (Millipore, MA, USA) and imaged using the LAS-4000 Gel Documentation System (FujiFilm, Tokyo, Japan). Densitometric analysis to quantify relative protein expression was carried out using ImageJ software. Coimmunoprecipitation (Co-IP) was carried out using protein lysates derived from BMMs. TCPs were extracted from cells with RIPA lysis buffer with protease and phosphatase inhibitor cocktail. Lysates were cleared, and $100 \mathrm{mg}$ of total protein were incubated at $4^{\circ} \mathrm{C}$ with $5 \mathrm{mg}$ of specific antibodies and $40 \mathrm{ml}$ protein $\mathrm{G}$-sepharose beads (Thermo Fisher Scientific) overnight with gentle rotation. Bead-antibody complexes were washed 3 times with lysis buffer and then boiled for 10 min to obtain protein supernatant captured by the protein $\mathrm{G}$-sepharose beads. The bound proteins were visualized by Western blot with corresponding antibody as previously described

\section{NF-кB p65 immunofluorescence staining}

The effect of CSB6B on NF-кB p65 nuclear translocation was examined using immunofluorescence analysis. Briefly, BMMs were pretreated with $20 \mu \mathrm{M}$ CSB6B for 30 mins followed by stimulation with $50 \mathrm{ng} / \mathrm{ml}$ RANKL for $30 \mathrm{mins}$. Cells were washed briefly, fixed in $4 \%$ PFA, and then permeabilized with $0.25 \%$ Triton X-100 for 5 mins. 
Cells were then incubated with monoclonal anti-p65 antibody in 2\% BSA-PBS for 2

271 hours at room temperature and then with Alexa Fluor 546 secondary antibody (Thermo

272 Fisher Scientific) for 1 hour at $4^{\circ} \mathrm{C}$ in the dark. Cell nucleus were counterstained with

273 DAPI (Santa Cruz Biotechnology) for 5 mins in the dark. Fluorescence images were

274 acquired on the Nikon A1 confocal laser microscope using the associated software

275 (Nikon, Tokyo, Japan). ImageJ was used to quantify the percentage of nuclear p65276 positive cells.

\section{Ti particle-induced calvarial osteolysis mode}

278 The in vivo effect of CSB6B on inflammation-induced bone loss was assess using the 279 murine titanum (Ti) particle-induced calvarial osteolysis model. 24 8-week old C57BL/6 mice were randomly divided into 4 groups ( $n=6$ per group): Sham-operated group (with PBS injection), vehicle group (Ti particles with PBS injection), low-dose group (Ti particles with $2 \mathrm{mg} / \mathrm{kg}$ CSB6B injection), and high-dose group (Ti particles with $8 \mathrm{mg} / \mathrm{kg}$ CSB6B injection). Briefly, $30 \mathrm{mg}$ of sterilized Ti particles were embedded under the cranial periosteum near the calvarial midline suture. Two days post-surgery, subcutaneous injections of CSB6B or PBS was carried out every 2 days for 2 weeks. At the end of the experimental period, all mice were sacrificed, the calvarial bones were removed, fixed in 4\% PFA and processed for micro-computed tomography $(\mu \mathrm{CT})$ and histological assessments. Ti particles were removed prior to $\mu \mathrm{CT}$ scanning to reduce metal artifact interference.

\section{Ovariectomy (OVX)-induced osteoporosis model}

The in vivo effect of CSB6B on post-menopausal bone loss was examined using the well-established murine model of OVX-induced bone loss in 8-week-old female C57BL/J6 mice. 24 mice were randomly assigned to 4 groups ( $n=6$ per group): Shamoperated group (injected with PBS), Vehicle group (OVX with PBS injection), lowdose group (OVX with injection of $2 \mathrm{mg} / \mathrm{kg}$ CSB6B), and high-dose group (OVX with injection of $8 \mathrm{mg} / \mathrm{kg} \mathrm{CSB6B}$ ). Mice underwent bilateral ovariectomy to remove ovaries and associated fallopian tubes (OVX groups) or sham operation. Four week postsurgery, mice received either intraperitoneal injections of CSB6B or equal volume of PBS every other day for 4 weeks. At the end of the experimental period, all mice were 
euthanized, and the tibiae and femurs were excised, fixed in 4\% PFA and processed for $\mu \mathrm{CT}$ and histological assessemnts. One set of of tibiae were grinded and subjected to RNA extraction using Trizol reagent (Thermo Fisher Scientific), and gene expression analysis was examined as described previously.

\section{$\mu C T$ scanning and analysis}

Fixed calvarial and long bone samples were subjected to $\mu \mathrm{CT}$ analysis using the Skyscan 1072 micro-CT system (Bruker, Billerica, MA, USA). Images were acquired with the scanning parameters set at a current of $80 \mathrm{~mA}$ and voltage of $70 \mathrm{kV}$ with an isometric pixel size of $9 \mathrm{~mm}$. Three-dimensional reconstructions of the bone tissues were carried out using the SkyScan NRecon software and morphometric analysis conducted using the SkyScan CTAn software (Bruker). For the long bones, a square region of interest $0.5 \mathrm{~mm}$ below the growth plate was defined for further quantitative analyses. For calvarial bones, a square region of interest around the midline suture was used for quantitative measurements. Bone morphometric parameters analyzed include bone volume/tissue volume (BV/TV), percentage of total porosity, mean trabecular thickness (Tb.Th, mm), mean trabecular separation (Tb.Sp, mm), and mean trabecular number (Tb.N, $\left.\mathrm{mm}^{-1}\right)$.

\section{Histology and histomorphometry}

Following $\mu \mathrm{CT}$ scanning, bone tissues were decalcified in $12.5 \%$ EDTA for 1 week at $4^{\circ} \mathrm{C}$ and then embedded into paraffin blocks. Histological sections of $5 \mu \mathrm{m}$ thick were prepared and then stained with hematoxylin and eosin (H\&E) and TRAP. Bone histomorphometric analyses on tissue sections were performed in a blinded, unbiased manner using ImageProPlus 6.0 software (Media Cybernetics, Rockville, MD, USA). The number of $\mathrm{TRAP}^{+}$osteoclasts were quantified and normalized to the bone area in each sample. To evaluate the effect of CSB6B on osteoblast bone formation in vivo, immunofluorescence staining for Runx2 was carried out. Sections were first blocked with goat serum and then incubated with anti-Runx 2 antibodies overnight at $4^{\circ} \mathrm{C}$. Sections were washed extensively but gently, then incubated with TRITC-conjugated secondary antibodies for 1 hour at room temperature in the dark, and finally with DAPI for 15 mins at room temperature in the dark. Fluorescence images were acquired on a 
Nikon A1 confocal microscope equipped with a digital camera.

\section{Bone serum markers}

Specific ELISA kits were used to detect serum bone turnover markers including mouse carboxy-terminal collagen crosslinks (CTX-1; Cusabio Technology LLC, Wuhan, China) and mouse procollagen type I intact N-terminal propeptide (P1NP; Cusabio Technology LLC).

\section{Statistical analysis}

Graphical results are presented as the means \pm SD of at least 3 independent experiments unless otherwise stated. Microscopic images are relevant representative images of experimental and control conditions. Statistical difference were determined using Student's $t$-test or one-way ANOVA followed by Tukey's post hoc analysis using Prism 7 software (GraphPad, San Diego, CA, USA). P-values less than 0.05 or unless otherwise indicated were considered statistically significant.

\section{Results}

\subsection{CSB6B inhibits RANKL-induced osteoclast differentiation and bone} resorption in vitro

The cellular cytotoxicity of CSB6B on BMMs was first assessed using the CCK-8 assay (Figure 1B). As shown in Figure 1B, concentrations of up to $25 \mu \mathrm{M}$ of CSB6B exhibited no cytotoxic effect on BMMs when treated for 48 and 96 hours. Dose-dependent decrease in cell viability was observed at concentrations of 50, 100 and $200 \mu \mathrm{M}$. As BMM-derived osteoclasts requires on average a minimum of 5 days to form following RANKL stimulation, sub-lethal concentrations $(5,10$, and $20 \mu \mathrm{M})$ of CSB6B was selected for subsequent investigations. The IC50 for BMMs was calculated to be 107.8 $\mu \mathrm{M}$ (Figure 1C). M-CSF-dependent BMMs were stimulated with RANKL without or with CSB6B for 5 days and the resulting osteoclasts were fixed and stained for TRAP activity. Compared with the large and well spread $\mathrm{TRAP}^{+}$multinucleated osteoclasts seen in RANKL-only treated controls, $\mathrm{TRAP}^{+}$cells in CSB6B treated groups were markedly smaller with the majority of the population being mononuclear cells (particularly when treated with 10 and $20 \mu \mathrm{M}$ CSB6B) (Figure 1D). This observation was further confirmed by quantitative assessments. As shown in Figure 1E, CSB6B not 
only reduced the total number of osteoclasts formed, but also dose-dependently inhibited the multi-nucleation and consequently the size of the osteoclasts that formed. These results suggests that CSB6B treatment affects mononuclear cell fusion, a process that is essential for the multi-nucleation characteristic of mature osteoclast. We further confirmed this observation by examining the actin cytoskeleton. BMM-derived osteoclasts were stained with rhodamine-conjugated phalloidin and the actin cytoskeleton examined under fluorescence microscopy. As shown in Figure 1F and G, the podosomal actin belt that demarcate individual osteoclasts were significantly smaller in CSB6B treatment groups than RANKL-only treated controls. Similarly, control osteoclasts exhibited numerous nuclei (DAPI stained; blue), whereas cells treated with either 10 or $20 \mu \mathrm{M}$ of CSB6B remained predominantly as mononuclear cells. In line with this inhibitory effect on osteoclast formation, the expression of gene required for osteoclast fusion and differentiation such as DC-STAMP, NFATc1, and cFos were markedly downregulated following CSB6B treatment (Figure 1H).

In addition to downregulation of genes involved in osteoclast fusion and differentiation, CSB6B also downregulated the expression of genes required for osteoclast bone resorption including TRAP, TCIRG1, and CTSK (Figure 1H). To confirm that the bone resorptive function was indeed impaired, we carried out bone resorption assays. Osteoclasts cultured on bovine bone discs and treated with CSB6B indeed showed markedly reduced ability to perform bone resorptive function (Figure 1I). Compared to the numerous resorption pits and trails seen in the control osteoclasts, a greater than $50 \%$ reduction in resorption pits were observed in CSB6B treated cells (Figure 1I). Resorption pits were scarce and randomly scattered around the bone discs (Figure 1J). Collectively these results provided evidence for the inhibitory effect of CSB6B on osteoclast formation and bone resorption in vitro.

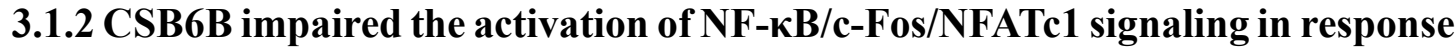
to RANKL

The transcription factors c-Fos and NFATc1 are crucial for osteoclasts differentiation, with the latter being the master transcription factor for various osteoclast marker genes[15-18]. As is shown in the Figure 2A-C, CSB6B treatment time- (Figure 2A) and 
dose-dependently (Figure 2B) attenuated the protein expression of both c-Fos and NFATc1. Given that the expression of c-Fos precedes and is required for the subsequent induction of NFATc1, the attenuation of NFATc1 observed here is likely the outcome of decreased c-Fos expression. As shown in Figure 1D and E, we found the protein expression of MIF was increased in M-CSF-dependent BMMs stimulated with RANKL 3 days and the MIF expression was decreased after the CSB6B treated. To further determine MIF protein metabolism following CSB6B treatment, cells were further treated with cycloheximide $(\mathrm{CHX})$ or MG132 respectively7. Treatment with the $26 \mathrm{~S}$ proteasome inhibitor MG132 partially restores MIF protein expression, whereas treatment with $\mathrm{CHX}$ which inhibits new protein biosynthesis further augmented the effects of CSB6B (Figure 2F and G). Furthermore, by co-IP analysis, we found that CSB6B treatment significantly increased the ubiquitination of MIF (Figure 2H). Together, these results suggests that the increased MIF protein degradation is responsible for the decreased expression of MIF protein following CSB6B treatment during RANKL-induced osteoclast formation.

The induction of c-Fos and NFATc1 requires the activation of early RANKL-induced signaling pathways. Of the many pathways that are activated, NF- $\kappa \mathrm{B}$ signaling is rapidly activated following RANKL signaling. RANKL-induced activation of NF- $\kappa B$ involves rapid degradation of $\mathrm{I} \kappa \mathrm{B} \alpha$ (within 10 mins), and subsequent phosphorylation and nuclear translocation of NF- $\kappa$ B p65 subunit (Figure 2I-L). CSB6B treatment on the other hand, diminished I $\mathrm{KB} \alpha$ and $\mathrm{p} 65$ phosphorylation, which consequently decreased the number of nuclear p65 (Figure 2I-L). Collectively these results suggests the antiosteoclastogneic and anti-resorptive effects of CSB6B can in part be attributed to the impairment of RANKL-induced activation of NF- $\kappa$ B/c-Fos/NFATc1 signaling cascades.

\subsubsection{CSB6B enhanced osteogenesis via suppression of NF-אB and induction of}

\section{Runx2}

Having now shown that CSB6B exerts inhibitory effects on osteoclast formation and activity we next examined whether CSB6B similarly affects osteoblasts given that osteoblast also expresses MIF. The effects of CSB6B on the viability of primary calvarial osteoblast were examined using CCK-8 assay. As with primary BMMs, 
CSB6B did not affect the viability of calvarial osteoblasts at concentrations of $25 \mu \mathrm{M}$ and below. However a dose- and time-dependent decrease in cell viability at concentrations of $50 \mu \mathrm{M}$ and above (Figure 3A). The IC50 for primary calvarial osteoblasts was calculated to be $61.93 \mu \mathrm{M}$ (Figure 3B). Once again, sub-lethal concentrations $(5,10$, and $20 \mu \mathrm{M})$ of CSB6B was used of osteogenic differentiation which can last for 7 days for ALP activity of 21 days for bone mineralization nodule formation. To examine the effects of CSB6B on osteoblast differentiation, calvarial osteoblast were cultured under osteogenic conditions without or with CSB6B for 7 days after which cells were fixed and stained for ALP activity. As is shown in Figures 3C and D, CSB6B significantly enhanced the osteogenic differentiation of calvarial osteoblasts as compared to untreated cells (Figure 3C). CSB6B more than doubled the number of $\mathrm{ALP}^{+\mathrm{ve}}$ cells when compared to untreated cells, but no significant difference in ALP activity was observed between the different concentrations of CSB6B (Figure 3D). For mineralization activity, calvarial osteoblasts were cultured under osteogenic conditions without or with CSB6B for 21 days. Cells were then fixed and stained with Alizarin Red S (ARS) to examine mineralized bone nodule formation. In the same manner as ALP activity, CSB6B markedly elevated the mineralization function of calvarial osteoblasts as compared to untreated cells (Figure 3C and D). Quantitative measurement of mineralized content confirmed the elevated bone formation activity following CSB6B treatment but not difference was observed between the various concentrations of CSB6B tested (Figure 3D). The elevated osteoblast formation and bone formation activity was confirmed by gene expression analyses. At 7 days postosteogenic differentiation, the expression of $A L P$ and $O C N$ was upregulated following CSB6B treatment in a dose-dependent matter (Figure 3E). Interestingly no effects on Runx 2 and $O P N$ gene expression was observed. On the other hand, at 21 days postosteogenic differentiation, the gene expression of Runx2, OCN, and $O P N$ were markedly upregulated whereas $A L P$ was downregulated (Figure 3F). Consistent with the upregulation in Runx2 gene expression 21 days post-osteogenic differentiation, the protein levels of Runx2 was also increased following CSB6B treatment (Figure 3G and $\mathrm{H})$. 
It is well established that NF- $\mathrm{BB}$ signaling negatively regulates osteoblast differentiation in part by interfering with Runx 2 expression and transcriptional activity [19-23]. Since NF- $\kappa B$ signaling was inhibited in osteoclasts following CSB6B treatment, we examined whether similar effects was also true in osteoblasts. As expected treatment of primary calvarial osteoblasts cultured under osteogenic media with CSB6B significantly inhibited the activation phosphorylation of p65 which suggests that the activation of canonical NF- $\mathrm{BB}$ was suppressed. Thus the stimulatory effect of CSB6B on osteoblast differentiation and mineralization can be in part attributed to elevated Runx2 expression and reduced NF- $\mathrm{kB}$ signaling activation.

\subsubsection{BMMs from MIF KO mice show decreased osteoclastogenic potential}

We have previously established that the genetic knockout of MIF decreased the osteoclastogenic potential of BMMs [13]. We used this MIF KO mouse line we examined whether CSB6B can further augment the cellular effects of the genetic loss of MIF. As is shown in the Figure 4A and B, CSB6B treatment did not further inhibit the formation of multinucleated $\mathrm{TRAP}^{+}$osteoclast from KO BMMs. Consistent with the effects of CSB6B treatment, KO BMMs exhibited impaired p65 phosphorylation and nuclear localization following RANKL stimulation (Figure 4C and D). Again treatment with CSB6B did not further enhance the inhibition of $\mathrm{p} 65$ phosphorylation following RANKL stimulation (Figure 4F-G). Similar results were seen with calvarial osteoblasts. Primary KO calvarial osteoblasts exhibited markedly stronger osteogenic potential than WT calvarial cells but this was not further enhanced by CSB6B treatment in BMMs and calvarial osteoblasts following CSB6B treatment was specifically via the inhibition of MIF and not off-target effects.

\subsubsection{In vivo administration of CSB6B prevents Ti particle-induced inflammatory} osteolysis

With promising in vitro cellular effects, we next investigated the potential protective effects of CSB6B treatment in pathological inflammatory bone destruction induced by Ti particles. Sterilized Ti particles were implanted under the calvarial periosteum of mice to induce localized inflammatory osteolysis. Mice were then either given 
subcutaneous injections of PBS (vehicle), or low-dose (2 mg/kg) CSB6B, or high-dose $(8 \mathrm{mg} / \mathrm{kg}$ ) dose CSB6B every 2 days for 4 weeks. As shown in the 3D reconstructions of $\mu \mathrm{CT}$ scans, CSB6B markedly alleviated inflammatory bone destruction and porosity induced by the Ti particles (Figure 5A and B). In comparison, mice treated with PBS vehicle exhibited severe osteolysis, extensive bone erosion, and marked elevated in bone porosity (Fgiure 5A and B). Histological H\&E examination showed extensive inflammatory response and bone destruction, whilst TRAP-stained sections revealed marked elevation in osteoclasts on bone surface. Treatment with CSB6B dosedependently attenuated inflammatory bone loss by reducing the number of TRAP ${ }^{+}$ osteoclasts on the bone surface (Figure 5C). Furthermore, by fluorescence immunostaining we found elevated expression of Runx2 lining the calvarial bone surface in mice treated with CSB6B suggesting elevation in osteoblast formation and or activity. Thus together, these data suggests that CSB6B treatment can protect mice against pathological inflammatory bone destruction induced by Ti particle via the inhibition of osteoclast formation and activity, and elevation of osteoblast formation.

\subsubsection{CSB6B protects against post-menopausal bone loss induced by ovariectomy} (OVX)

With the encouraging protective effects against pathological bone destruction, we next examined whether CSB6B can also protect against post-menopausal bone loss induced by OVX. Four weeks after bilateral ovariectomy, mice were treated with intraperitoneal injections of PBS, low-dose, or high-dose CSB6B for further 4 weeks. At the end of the experimental period, the left tibia were harvested and processed for $\mu \mathrm{CT}$ analysis. As shown in Figure 6A and B, CSB6B treatment protected mice against OVX-induced bone loss in a dose-dependent manner improving bone morphometric parameters of BV/TV, Tb.Th (mm), Tb.N ( $\left.\mathrm{mm}^{-1}\right)$, and Tb.Sp (mm) to near sham levels. Histological $\mathrm{H} \& \mathrm{E}$ analysis further confirmed the protective effects of CSB6B against OVX-induced bone loss, with TRAP stained sections further revealing marked reduction in the number of $\mathrm{TRAP}^{+}$osteoclasts on the trabecular bone surface (Figure 6C and D). Reduction in osteoclast activity was further confirmed by serum CTX-1 measurements, with lowdose CSB6B treatment reducing bone resorption activity to sham levels and high-dose 
reducing it further below sham levels (Figure 6E). Gene expression analysis using RNA extracted from the tibial bone tissues further confirmed the reduction in osteoclast formation and activity following CSB6B treatment. The gene expression of NFATc1, CTSK, and TRAP were noticeably downregulated in most cases back down to sham levels (Figure 6F). Thus these results clearly indicate that in vivo administration of CSB6B treatment can effectively inhibit the elevated osteoclast formation and bone resorption induced by OVX.

Next we examined whether similar favourable osteoblastic effects were seen. Immunofluorescence staining of long bone sections for Runx2 again revealed elevated expression of Runx 2 on the trabecular bone surface in CSB6B treated mice as compared to vehicle control suggesting increased osteoblast formation (Figure 7A and B). Furthermore, analysis of serum P1NP levels, a measure of osteoblast bone formation activity, was evidently increased following CSB6B treatment (Figure 7C). Furthermore expression of osteoblastic marker genes (Runx2, $A L P, O C N$, and $O P N)$ were in general (particularly at high-dose) upregulated following CSB6B treatment further reinforcing the notion that osteoblast formation and activity were increased (Figure 7D). Thus collectively, we observed potent protective effect of CSB6B against OVX-induced bone loss again via the suppression of osteoclast formation and bone resorption, and elevation in osteoblast bone formation.

\section{Discussion}

MIF is a unique pleiotropic pro-inflammatory mediator expressed in numerous immune cell including monocytes/macrophages and plays an important role in the innate immune response[24]. MIF not only functions as a chemoattractant for inflammatory cells, it also acts to attract osteoclast precursors to osteolytic lesion enhancing osteoclast-mediated bone destruction[25]. MIF expression is upregulated during RANKL-induced osteoclast differentiation and numerous other studies including our own have shown direct and indirect stimulatory effect of MIF on osteoclast formation and bone resorption[10,26-29]. Thus inhibition of MIF activity is an attractive therapeutic option for the mitigation of elevated bone resorption in various bone diseases. In our study, we showed that Chicago Sky Blue 6B (CSB6B), a unique inhibitor of MIF, exerts 
540 potent inhibitory effect on osteoclast formation and bone resorption, as well as

541 stimulatory effects on osteoblast bone formation in vitro. Administration of CSB6B also

542 protects mice against pathological inflammatory bone destruction induced by $\mathrm{Ti}$

543 particles, and estrogen-deficiency induced bone loss due to OVX. This in vivo

544 therapeutic effect of CSB6B aligned with previous studies which showed that MIF KO

545 mice were resistant to OVX-induced bone loss and MIF transgenic mice exhibited high

546 turnover osteoporosis [11,12].

547 CSB6B is a unique sulfonated azo compound that allosterically binds at the interface

548 of two MIF trimers to block the cytokine and catalytic activities of MIF [14]. Here in

549 this study, using primary BMMs we showed that CSB6B treatment can inhibited

550 RANKL-induced osteoclast formation and function. This inhibitory effect of CSB6B

551 was consistent with impaired osteoclast formation derived from MIF KO BMMs.

552 Furthermore, CSB6B treatment of MIF KO BMMs did not further enhance the

553 inhibitory effect implying that the anti-osteoclastic effect of CSB6B is specifically

554 through the inhibition of MIF. Additionally, our findings were in line with other studies

555 using MIF KO BMMs that showed similar defects in osteoclast formation and bone

556 resorption[26,29]. We have also previously shown that 4-IPP, a suicide substrate for MIF

557 that inhibits MIF activity, inhibits RANKL-induced osteoclast formation and bone

558 resorption[13]. Thus our studies and others have provided evidence that MIF positively

559 involved in osteoclast formation and activity, and pharmacological inhibition of MIF

560 offers alternative therapeutic option against osteoclast-mediated bone loss.

561 Corroborating these effects, we further showed that the in vivo treatment of CSB6B

562 effectively protected mice against inflammatory calvarial bone destruction induced by

563 Ti particles as well as estrogen-deficiency induced bone loss as a result of OVX. We

564 showed reduced levels of serum CTX-1, a marker of bone resorption, indicating that

565 CSB6B treatment reduced osteoclast-mediated bone resorption.

566 Mechanistically, RANKL-induced osteoclast differentiation requires the early

567 activation of several key signaling cascades. Among these the NF- $\kappa \mathrm{B}$ signaling pathway

568 is crucial for the downstream induction of various transcription factors that are crucial

569 for osteoclast differentiation such as c-Fos and NFATc1 [30]. In unstimulated cells, the 
$570 \mathrm{NF}-\kappa \mathrm{B}$ transcription factor consisting of p65 and p50 heterodimeric complex are 571 retained in the cytoplasm by NF- $\kappa$ B inhibitory proteins, called I $\kappa$ Bs $[31,32]$. Upon 572 stimulation by RANKL IкBs are phosphorylated by the IKK complex (consisting of $573 \mathrm{IKK} \alpha$, IKK $\beta$, and IKK $\gamma$ ) which consequently leads to IKB degradation via the $26 \mathrm{~S}$ 574 proteasome. This then allows the free NF- $\mathrm{kB}$ p65/p50 dimers to be phosphorylated 575 resulting in its nuclear translocation where it transcriptional regulated the expression of 576 downstream target genes necessary for osteoclast differentiation including c-Fos and 577 NFATc1 $[30,33,34]$. In our biochemical analyses we found that RANKL-induced p65 578 phosphorylation was significantly reduced following CSB6B treatment. In our previous 579 study, we found using co-IP that MIF interacts with TXNIP and p65 and that this interaction is necessary for p65 phosphorylation and nuclear translocation. Thus the reduced p65 phosphorylation and nuclear translocation following CSB6B treatment could in part due to inhibition of MIF/TXNIP/p65 interaction. Further investigation will need to be carried out to ascertain this effect.

The induction of transcription factors c-Fos and NFATc1 is downstream of NF-kB signaling activation. c-Fos is component of the AP-1 transcription factor complex that synergises with NF- $\mathrm{kB}$ for initial induction of NFATc1, the key transcriptional regulator of osteoclast formation. Subsequent sustained induction and activation of NFATc1 is achieved by NFATc1 auto-amplification triggered by calcium signaling. This sustained induction of NFATc1 activity is necessary for transcriptional upregulation of numerous osteoclast marker genes [15,35]. Genetic disruption of c-Fos or NFATc1 leads to severe osteopetrotic phenotypes in mice highlighting the importance of these transcription factors in osteoclasts biology [15,36-38]. We showed that MIF neutralization with CSB6B markedly reduced the induction of both c-Fos and NFATc1 proteins which consequently downregulated the expression of osteoclast marker genes DC-STAMP, TRAP, TCIRG1 and CTSK which are under the control of NFATc1.

To some extent the reduced induction of these transcription factors could be explained by impaired NF- $\kappa \mathrm{B}$ signaling activation. However, we demonstrated that additional mechanism of increased MIF proteasomal degradation induced by CSB6B was involved in the reduction in MIF protein levels. At the protein level, MIF could 
ubiquitylated for targeting to proteasome for degradation [39]. Here we used cycloheximide (CHX) and MG132 to demonstrate that CSB6B the reduced protein expression of MIF was due to increased MIF ubiquitylation and proteasomal degradation. Previous studies have shown that the HSP90-associated E3 ubiquitin ligase CHIP mediates the ensuing proteasome-dependent MIF degradation[39], but whether the effect of CSB6B on MIF degradation is CHIP-dependent or independent will require further intricate studies to be conducted.

While MIF is also expressed in osteoblastic cells, the biological effects of MIF on osteoblasts are not as well-defined. Studies have suggested that MIF do no exert any biological activities on osteoblast formation or differentiation whilst others suggest proosteoblastic effects $[12,28,40-42]$. Contrary to these studies, we have found that inhibition of MIF by CSB6B significantly enhanced osteoblast differentiation and mineralized bone nodule formation in vitro, supported by upregulation of steoblast marker gene expression such as Runx2, $A L P, O C N$, and $O P N$. Importantly Runx2 is a transcription factor required for osteoblast differentiation and maturation [43]. Interestingly, MIF have previously been shown to downregulate the gene expression of Runx 2 by indirectly regulating the transcriptional activation of Runx2 promoter [44]. The result by Yao and colleagues is line with our findings in this paper and previous paper [13] suggesting that MIF may negatively regulate osteoblast differentiation and bone formation activity. Additionally inhibition of $\mathrm{NF}-\kappa \mathrm{B}$ signaling could have also contributed to the stimulatory effect of CSB6B on osteoblast differentiation and activity. $\mathrm{NF}-\kappa \mathrm{B}$ has been shown to target the Runx2 promoter to inhibit its activation [45]. We showed that p65 phosphorylation was markedly reduced following CSB6B treatment. Thus the MIF/NF- $\kappa \mathrm{B} / \mathrm{Runx} 2$ may function as a novel negative regulatory mechanism for osteoblast differentiation and bone formation. In accordance with our in vitro results, increased bone formation activity in vivo was also noted histologically and by analysis of serum levels of P1NP. This positive effect on osteoblast bone formation no doubt contributed to the protective effect of CSB6B against both Ti-particle induced calvarial osteolysis and OVX-induced bone loss. 
In conclusion, our study have provided convincing evidence that CSB6B inhibits osteoclast formation and bone resorption via the suppression of NF- $\mathrm{kB}$ activation and increased proteasomal degradation of MIF. Additionally, CSB6B exerts proosteoblastic effects via the attenuation of MIF/NF- $\mathrm{BB}$ inhibitory action on Runx2 expression. By exerting both anti-osteoclastic and pro-osteoblastic effects, the MIF inhibitor CSB6B effectively protected mice against inflammatory bone destruction and estrogen-deficiency induced bone loss. Hence, we believe that CSB6B can be used as candidate inhibitor for the development of novel therapeutic agents against MIF for the treatment of osteolytic conditions.

\section{Conclusion:}

Together, as an MIF inhibitor, CSB6B can inhibit osteoclast differentiation and absorption function and enhance the mineralization of osteoblasts through the inhibition of NF- $\kappa$ B pathway. In vivo, CSB6B can relieve cranial lysis caused by Ti particles and osteoporosis induced by ovariectomy. Therefore, MIF is a prime target for therapeutic targeting for the treatment of osteolytic bone disorders and the MIF inhibitor CSB6B could be a potential anti-osteoporosis drug.

\section{Abbreviations:}

CSB6B, Chicago Sky Blue 6B; MIF, macrophage migration inhibitory factor; RANKL, receptor activator of nuclear factor- $\mathrm{kB}$ ligand; TCP: Total cellular proteins; NFATc1, nuclear factor of activated T cells cytoplasmic 1; KO, knockout; FBS, fetal bovine serum; DMEM, Dulbecco's Modified Eagle Medium; $\alpha$-MEM, alpha modification of Eagle's medium; TRAP, tartrate-resistant acid phosphatase; BMMs, bone marrow monocytes/macrophages; PFA, paraformaldehyde; ALP, alkaline phosphatase; OD, optical density; CCK-8, cell counting kit-8; qPCR, real-time quantitative PCR; OVX, ovariectomy; BV/TV, bone volume/tissue volume; Tb. Th, 
mean trabecular thickness; $\mathrm{Tb}$. Sp, mean trabecular separation; Tb. N, mean trabecular number; CTX-1, carboxy-terminal collagen crosslinks; P1NP, procollagen type I intact N-terminal propeptide; IC50, the half maximal inhibitory concentration; Ubi, ubiquintin; OCN, osteocalcin; OPN, osteopontin;

\section{Declarations:}

\section{Ethical Approval and Consent to participate}

Not applicable

\section{Consent for publication}

Not applicable.

\section{Availability of data and material}

The datasets used and/or analysed during the current study are available from the corresponding author on reasonable request.

\section{Competing interests}

The authors declare no conflict of interest

\section{Funding}

This work was funded by grants from the Zhejiang Province Scientific Project of Health and Medicine of China (Grant No.2018KY936), the Zhejiang Experimental Animal and Technology Program Foundation of China (Grant No.2018C37099) and the Medical key (support) discipline construction project of China (Grant No.2017ZDXK06). The authors declare no conflicts of interest.

\section{Author's contributions}

Kangtao Jin, Zhenzhong Chen, and Dengwei He designed the research; Kangtao Ji,Lin Zheng, Ziang Xie, Lin Ye, Jiawei Gao, Chao Lou, Wenzheng Pan performed the experiments; Kangtao Jin, Bin Pan, Shijie Liu analyzed the data; Kangtao Jin, Lin 
Zheng and Dengwei He wrote the manuscript. All authors read and approved the final manuscript.

\section{Acknowledgments}

This work was supported by grants from the Zhejiang Province Scientific Project of Health and Medicine of China (Grant No.2018KY936), the Zhejiang Experimental Animal and Technology Program Foundation of China (Grant No.2018C37099) and the Medical key (support) discipline construction project of China (Grant No.2017ZDXK06). The authors declare no conflicts of interest.

\section{Author details}

${ }^{1}$ Department of Orthopedics, Affiliated Lishui Hospital of Zhejiang University/the Fifth Affiliated Hospital of Wenzhou Medical University/Lishui Central Hospital, Lishui, China. ${ }^{2}$ Department of Orthopaedic Surgery, Sir Run Run Shaw Hospital, Zhejiang University School of Medicine, Hangzhou, China. ${ }^{3}$ Key Laboratory of Musculoskeletal System Degeneration and Regeneration Translational Research of Zhejiang Province, Hangzhou, China.

\section{REFERENCES}

1. Hadjidakis, D.J.; Androulakis, II. Bone remodeling. Ann N Y Acad Sci 2006, 1092, 385-396, doi:10.1196/annals.1365.035.

2. Crockett, J.C.; Rogers, M.J.; Coxon, F.P.; Hocking, L.J.; Helfrich, M.H. Bone remodelling at a glance. J Cell Sci 2011, 124, 991-998, doi:10.1242/jcs.063032.

3. Feng, X.; McDonald, J.M. Disorders of bone remodeling. Annu Rev Patho/ 2011, 6, 121 145, doi:10.1146/annurev-pathol-011110-130203.

4. Ilyas, Z;; Camacho, P.M. Rare adverse effects of bisphosphonate therapy. Curr Opin Endocrinol Diabetes Obes 2019, 10.1097/MED.0000000000000501, doi:10.1097/MED.0000000000000501.

5. David, J.R. Delayed hypersensitivity in vitro: its mediation by cell-free substances formed by lymphoid cell-antigen interaction. Proc Natl Acad Sci U S A 1966, 56, 72-77, doi:10.1073/pnas.56.1.72.

6. Calandra, T.; Bernhagen, J.; Metz, C.N.; Spiegel, L.A.; Bacher, M.; Donnelly, T.; Cerami, A.; 
Bucala, R. MIF as a glucocorticoid-induced modulator of cytokine production. Nature 1995, 377, 68-71, doi:10.1038/377068a0.

7. Calandra, T.; Bernhagen, J.; Mitchell, R.A.; Bucala, R. The macrophage is an important and previously unrecognized source of macrophage migration inhibitory factor. $J$ Exp Med 1994, 179, 1895-1902, doi:10.1084/jem.179.6.1895.

8. Singh, A.; Leng, L.; Fan, J.; Gajda, M.; Brauer, R.; Fingerle-Rowson, G.; Bucala, R.; Illges, H. Macrophage-derived, macrophage migration inhibitory factor (MIF) is necessary to induce disease in the K/BxN serum-induced model of arthritis. Rheumatol Int 2013, 33, 2301-2308, doi:10.1007/s00296-013-2713-4.

9. Chen, Z.; Ma, T.; Huang, C.; Zhang, L.; Hu, T.; Li, J. MIF, a potential therapeutic target for rheumatoid arthritis? Rheumatol Int 2014, 34, 1481-1482, doi:10.1007/s00296-0132877-y.

10. Madeira, M.F.; Queiroz-Junior, C.M.; Costa, G.M.; Santos, P.C.; Silveira, E.M.; Garlet, G.P.; Cisalpino, P.S.; Teixeira, M.M.; Silva, T.A.; Souza Dda, G. MIF induces osteoclast differentiation and contributes to progression of periodontal disease in mice. Microbes Infect 2012, 14, 198-206, doi:10.1016/j.micinf.2011.09.005.

11. Oshima, S.; Onodera, S.; Amizuka, N.; Li, M.; Irie, K.; Watanabe, S.; Koyama, Y.; Nishihira, J.; Yasuda, K.; Minami, A. Macrophage migration inhibitory factor-deficient mice are resistant to ovariectomy-induced bone loss. FEBS Lett 2006, 580, 1251-1256, doi:10.1016/j.febslet.2006.01.038.

12. Onodera, S.; Sasaki, S.; Ohshima, S.; Amizuka, N.; Li, M.; Udagawa, N.; Irie, K.; Nishihira, J.; Koyama, Y.; Shiraishi, A., et al. Transgenic mice overexpressing macrophage migration inhibitory factor (MIF) exhibit high-turnover osteoporosis. J Bone Miner Res 2006, 21 , 876-885, doi:10.1359/jbmr.060310.

13. Zheng, L.; Gao, J.; Jin, K.; Chen, Z.; Yu, W.; Zhu, K.; Huang, W.; Liu, F.; Mei, L.; Lou, C., et al. Macrophage migration inhibitory factor (MIF) inhibitor 4-IPP suppresses osteoclast formation and promotes osteoblast differentiation through the inhibition of the NFkappaB signaling pathway. FASEB J 2019, 33, 7667-7683, doi:10.1096/fj.201802364RR.

14. Bai, F.; Asojo, O.A.; Cirillo, P.; Ciustea, M.; Ledizet, M.; Aristoff, P.A.; Leng, L.; Koski, R.A.; Powell, T.J.; Bucala, R., et al. A novel allosteric inhibitor of macrophage migration inhibitory factor (MIF). J Biol Chem 2012, 287, 30653-30663, doi:10.1074/jbc.M112.385583.

15. Takayanagi, H.; Kim, S.; Koga, T.; Nishina, H.; Isshiki, M.; Yoshida, H.; Saiura, A.; Isobe, M.; Yokochi, T.; Inoue, J., et al. Induction and activation of the transcription factor NFATc1 (NFAT2) integrate RANKL signaling in terminal differentiation of osteoclasts. Dev Ce//2002, 3, 889-901.

16. Kim, K.; Kim, J.H.; Lee, J.; Jin, H.M.; Lee, S.H.; Fisher, D.E.; Kook, H.; Kim, K.K.; Choi, Y.; Kim, $\mathrm{N}$. Nuclear factor of activated $\mathrm{T}$ cells $\mathrm{c} 1$ induces osteoclast-associated receptor gene expression during tumor necrosis factor-related activation-induced cytokine-mediated osteoclastogenesis. J Bio/ Chem 2005, 280, 35209-35216, doi:10.1074/jbc.M505815200.

17. Sharma, S.M.; Bronisz, A.; Hu, R.; Patel, K.; Mansky, K.C.; Sif, S.; Ostrowski, M.C. MITF and PU.1 recruit p38 MAPK and NFATc1 to target genes during osteoclast differentiation. J Biol Chem 2007, 282, 15921-15929, doi:10.1074/jbc.M609723200.

18. Balkan, W.; Martinez, A.F.; Fernandez, I.; Rodriguez, M.A.; Pang, M.; Troen, B.R. Identification of NFAT binding sites that mediate stimulation of cathepsin $\mathrm{K}$ promoter 
activity by RANK ligand. Gene 2009, 446, 90-98, doi:10.1016/j.gene.2009.06.013.

19. Chang, J.; Wang, Z.; Tang, E.; Fan, Z.; McCauley, L.; Franceschi, R.; Guan, K.; Krebsbach, P.H.; Wang, C.Y. Inhibition of osteoblastic bone formation by nuclear factor-kappaB. Nat Med 2009, 15, 682-689, doi:10.1038/nm.1954.

20. Li, Y.; Li, A.; Strait, K.; Zhang, H.; Nanes, M.S.; Weitzmann, M.N. Endogenous TNFalpha lowers maximum peak bone mass and inhibits osteoblastic Smad activation through NFkappaB. J Bone Miner Res 2007, 22, 646-655, doi:10.1359/jbmr.070121.

21. Gilbert, L.; He, X.; Farmer, P.; Rubin, J.; Drissi, H.; van Wijnen, A.J.; Lian, J.B.; Stein, G.S.; Nanes, M.S. Expression of the osteoblast differentiation factor RUNX2 (Cbfa1/AML3/Pebp2alpha A) is inhibited by tumor necrosis factor-alpha. J Biol Chem 2002, 277, 2695-2701, doi:10.1074/jbc.M106339200.

22. Alles, N.; Soysa, N.S.; Hayashi, J.; Khan, M.; Shimoda, A.; Shimokawa, H.; Ritzeler, O.; Akiyoshi, K.; Aoki, K.; Ohya, K. Suppression of NF-kappaB increases bone formation and ameliorates osteopenia in ovariectomized mice. Endocrinology 2010, 151, 4626-4634, doi:10.1210/en.2010-0399.

23. Yamazaki, M.; Fukushima, H.; Shin, M.; Katagiri, T.; Doi, T.; Takahashi, T.; Jimi, E. Tumor necrosis factor alpha represses bone morphogenetic protein (BMP) signaling by interfering with the DNA binding of Smads through the activation of NF-kappaB. J Biol Chem 2009, 284, 35987-35995, doi:10.1074/jbc.M109.070540.

24. O'Reilly, C.; Doroudian, M.; Mawhinney, L.; Donnelly, S.C. Targeting MIF in Cancer: Therapeutic Strategies, Current Developments, and Future Opportunities. Med Res Rev 2016, 36, 440-460, doi:10.1002/med.21385.

25. Movila, A.; Ishii, T.; Albassam, A.; Wisitrasameewong, W.; Howait, M.; Yamaguchi, T.; RuizTorruella, M.; Bahammam, L.; Nishimura, K.; Van Dyke, T., et al. Macrophage Migration Inhibitory Factor (MIF) Supports Homing of Osteoclast Precursors to Peripheral Osteolytic Lesions. J Bone Miner Res 2016, 31, 1688-1700, doi:10.1002/jbmr.2854.

26. Howait, M.; Albassam, A.; Yamada, C.; Sasaki, H.; Bahammam, L.; Azuma, M.M.; Cintra, L.T.A.; Satoskar, A.R.; Yamada, S.; White, R., et al. Elevated Expression of Macrophage Migration Inhibitory Factor Promotes Inflammatory Bone Resorption Induced in a Mouse Model of Periradicular Periodontitis. J Immunol 2019, 202, 2035-2043, doi:10.4049/jimmunol.1801161.

27. Kim, H.R.; Kim, K.W.; Jung, H.G.; Yoon, K.S.; Oh, H.J.; Cho, M.L.; Lee, S.H. Macrophage migration inhibitory factor enhances osteoclastogenesis through upregulation of RANKL expression from fibroblast-like synoviocytes in patients with rheumatoid arthritis. Arthritis Res Ther 2011, 13, R43, doi:10.1186/ar3279.

28. Kobayashi, T.; Onodera, S.; Kondo, E.; Tohyama, H.; Fujiki, H.; Yokoyama, A.; Yasuda, K. Impaired fracture healing in macrophage migration inhibitory factor-deficient mice. Osteoporos Int 2011, 22, 1955-1965, doi:10.1007/s00198-010-1385-0.

29. Gu, R.; Santos, L.L.; Ngo, D.; Fan, H.; Singh, P.P.; Fingerle-Rowson, G.; Bucala, R.; Xu, J.; Quinn, J.M.; Morand, E.F. Macrophage migration inhibitory factor is essential for osteoclastogenic mechanisms in vitro and in vivo mouse model of arthritis. Cytokine 2015, 72, 135-145, doi:10.1016/j.cyto.2014.11.015.

30. Vaira, S.; Alhawagri, M.; Anwisye, l.; Kitaura, H.; Faccio, R.; Novack, D.V. RelA/p65 promotes osteoclast differentiation by blocking a RANKL-induced apoptotic JNK pathway in mice. $J$ 
Clin Invest 2008, 118, 2088-2097, doi:10.1172/JCI33392.

31. Vallabhapurapu, S.; Karin, M. Regulation and function of NF-kappaB transcription factors in the immune system. Annu Rev Immunol 2009, 27, 693-733, doi:10.1146/annurev.immunol.021908.132641.

32. Hayden, M.S.; Ghosh, S. Shared principles in NF-kappaB signaling. Ce// 2008, 132, $344-$ 362, doi:10.1016/j.cell.2008.01.020.

33. Boyce, B.F.; Yao, Z.; Xing, L. Functions of nuclear factor kappaB in bone. Ann N Y Acad SCi 2010, 1192, 367-375, doi:10.1111/j.1749-6632.2009.05315.x.

34. Blair, H.C.; Robinson, L.J.; Zaidi, M. Osteoclast signalling pathways. Biochem Biophys Res Commun 2005, 328, 728-738, doi:10.1016/j.bbrc.2004.11.077.

35. Yamashita, T.; Yao, Z.; Li, F.; Zhang, Q.; Badell, I.R.; Schwarz, E.M.; Takeshita, S.; Wagner, E.F.; Noda, M.; Matsuo, K., et al. NF-kappaB p50 and p52 regulate receptor activator of NF-kappaB ligand (RANKL) and tumor necrosis factor-induced osteoclast precursor differentiation by activating c-Fos and NFATc1. J Biol Chem 2007, 282, 18245-18253, doi:10.1074/jbc.M610701200.

36. Arai, A.; Mizoguchi, T.; Harada, S.; Kobayashi, Y.; Nakamichi, Y.; Yasuda, H.; Penninger, J.M.; Yamada, K.; Udagawa, N.; Takahashi, N. Fos plays an essential role in the upregulation of RANK expression in osteoclast precursors within the bone microenvironment. J Cell Sci 2012, 125, 2910-2917, doi:10.1242/jcs.099986.

37. Grigoriadis, A.E.; Wang, Z.Q.; Cecchini, M.G.; Hofstetter, W.; Felix, R.; Fleisch, H.A.; Wagner, E.F. C-Fos: a key regulator of osteoclast-macrophage lineage determination and bone remodeling. Science 1994, 266, 443-448, doi:10.1126/science.7939685.

38. Wang, Z.Q.; Ovitt, C.; Grigoriadis, A.E.; Mohle-Steinlein, U.; Ruther, U.; Wagner, E.F. Bone and haematopoietic defects in mice lacking c-fos. Nature 1992, 360, 741-745, doi:10.1038/360741a0.

39. Schulz, R.; Marchenko, N.D.; Holembowski, L.; Fingerle-Rowson, G.; Pesic, M.; Zender, L.; Dobbelstein, M.; Moll, U.M. Inhibiting the HSP90 chaperone destabilizes macrophage migration inhibitory factor and thereby inhibits breast tumor progression. J Exp Med2012, 209, 275-289, doi:10.1084/jem.20111117.

40. Ranganathan, V.; Ciccia, F.; Zeng, F.; Sari, I.; Guggino, G.; Muralitharan, J.; Gracey, E.; Haroon, N. Macrophage Migration Inhibitory Factor Induces Inflammation and Predicts Spinal Progression in Ankylosing Spondylitis. Arthritis Rheumato/ 2017, 69, 1796-1806, doi:10.1002/art.40175.

41. Onodera, S.; Suzuki, K.; Matsuno, T.; Kaneda, K.; Kuriyama, T.; Nishihira, J. Identification of macrophage migration inhibitory factor in murine neonatal calvariae and osteoblasts. Immunology 1996, 89, 430-435, doi:10.1046/j.1365-2567.1996.d01-751.x.

42. Onodera, S.; Nishihira, J.; Iwabuchi, K.; Koyama, Y.; Yoshida, K.; Tanaka, S.; Minami, A. Macrophage migration inhibitory factor up-regulates matrix metalloproteinase- 9 and 13 in rat osteoblasts. Relevance to intracellular signaling pathways. J Bio/ Chem 2002, 277, 7865-7874, doi:10.1074/jbc.M106020200.

43. Ducy, P.; Zhang, R.; Geoffroy, V.; Ridall, A.L.; Karsenty, G. Osf2/Cbfa1: a transcriptional activator of osteoblast differentiation. Cell 1997, 89, 747-754, doi:10.1016/s00928674(00)80257-3.

44. Yao, Y.; Deng, Q.; Song, W.; Zhang, H.; Li, Y.; Yang, Y.; Fan, X.; Liu, M.; Shang, J.; Sun, C., et 
al. MIF Plays a Key Role in Regulating Tissue-Specific Chondro-Osteogenic Differentiation Fate of Human Cartilage Endplate Stem Cells under Hypoxia. Stem Cell Reports 2016, 7 , 249-262, doi:10.1016/j.stemcr.2016.07.003.

45. Yao, Z.; Li, Y.; Yin, X.; Dong, Y.; Xing, L.; Boyce, B.F. NF-kappaB RelB negatively regulates osteoblast differentiation and bone formation. J Bone Miner Res 2014, 29, 866-877, doi:10.1002/jbmr.2108.

\section{Figures}

Fig.1

A

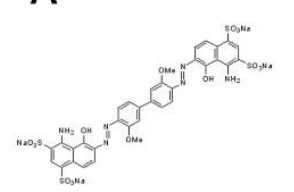

D
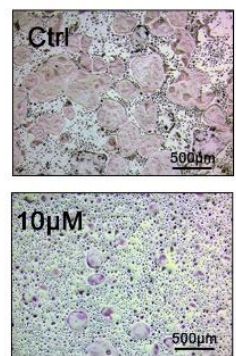

F

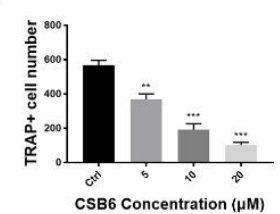

$\mathrm{H}$
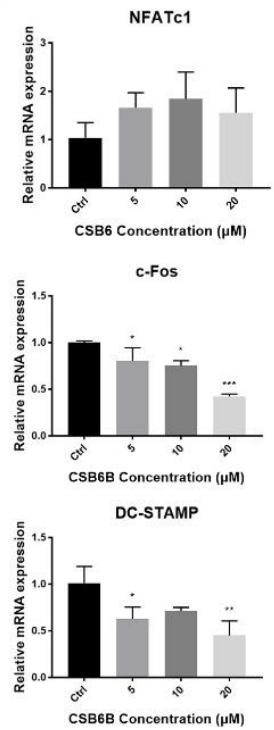

B
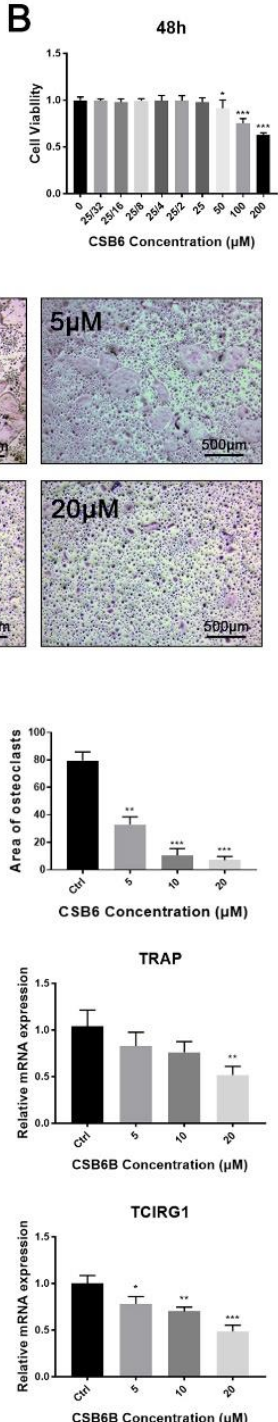

CTSK

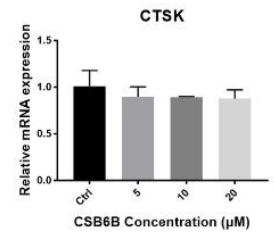

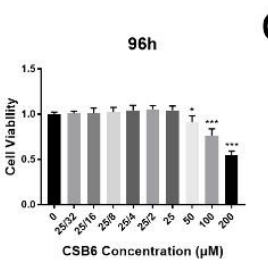

C

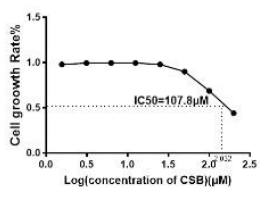

E
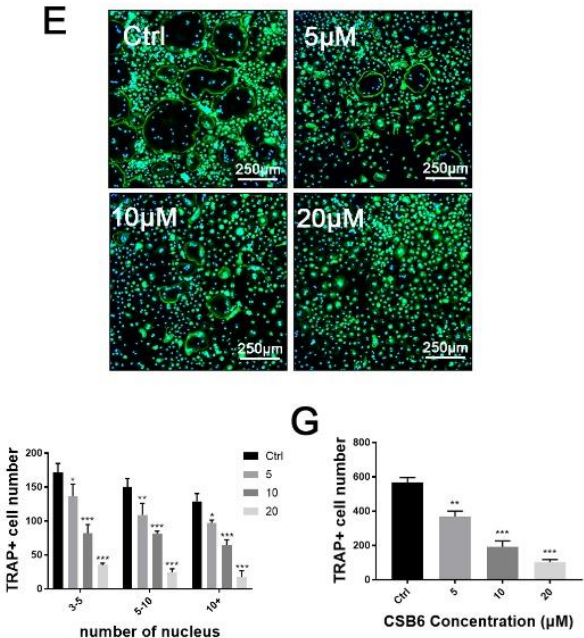

I

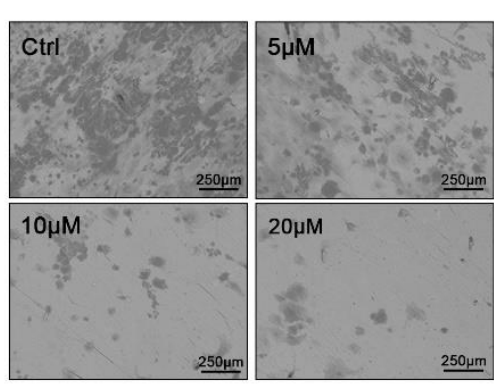

J

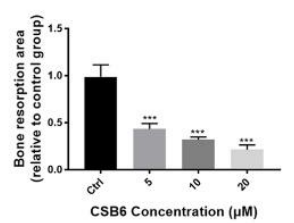


863

864

865

866

867

868

869

870

871

872

873

874

875

876

877

878

879

880

881

Fig 1. CSB6B inhibited osteoclast formation in a dose-dependent in vitro. A) The structure of CSB6B. B) Cytotoxic effects of CSB6B on BMMs were assessed by CCK8 cell viability/cytoxicity assay at 48 and 96 h. C) IC50 values obtained for the activity of CSB6B against BMMs. D) BMMs were treated with RANKL(50 ng/ml), M-CSF(40 $\mathrm{ng} / \mathrm{ml}$ ) without or with different concentrations (Con.) of CSB6B for $5 \mathrm{~d}$, after that osteoclasts were fixed and stained for TRAP. Scale bars, $500 \mu \mathrm{m}$. F) The number and size (mean area) of TRAP-positive multinucleated osteoclasts with $>5$ nuclei were quantified. The distribution of TRAP-positive osteoclasts with $3-5,5-10$, or $>10$ nuclei were also determined. E) Following treatment with indicated concentrations of CSB6B, actin belts were stained with rhodamine-conjugated phalloidin and analyzed by immunofluorescence microscopy. Nuclei were counterstained with DAPI. Scale bars, $250 \mu \mathrm{m}$. G) The number of actin belts in four groups were quantified. H) Expression of osteoclasts genes in BMMs simulated with RANKL and different concentration of CSB6B for 5d such as NFATc1, c-Fos, DC-STAMP, TRAP, TCIRG1 and CTSK were analyzed by qPCR. I) Bone resorptive activity of BMMs cultured on bovine bone discs were assessed by scanning electron microscopy following treatment with CSB6B $(20 \mu \mathrm{M})$. Scale bars, $250 \mu \mathrm{m}$. J) The mean resorption pit area was quantified. Images presented are representative of $\geq 3$ independent experiments, and data are expressed as means \pm SD. $* \mathrm{P} \leq 0.05, * * \mathrm{P} \leq 0.01, * * * \mathrm{P} \leq 0.005$ when compared with control group. 
Fig.2

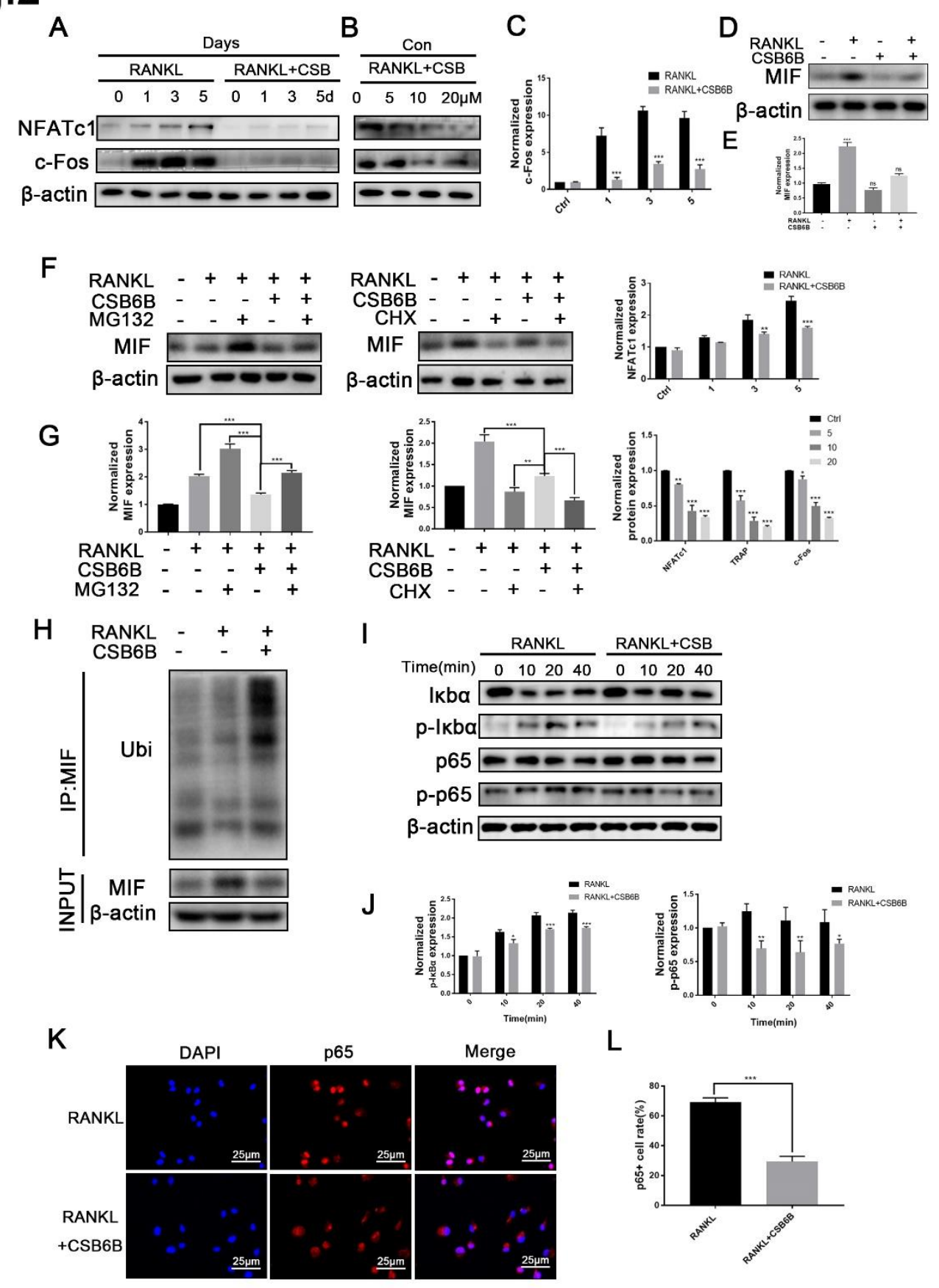

Figure 2

Fig2. CSB6B inhibits early NF-кB signaling. A, B) TCPs from BMMs stimulated with RANKL without or with CSB6B $(20 \mu \mathrm{M})$ for $0,1,3$, or $5 \mathrm{~d}$ (late time course) or with indicated concentrations of CSB6B (dose dependent) for $5 \mathrm{~d}$ were extracted and subjected to Western blot analysis with specific antibodies against NFATc1 and c-Fos. Expression of $\beta$-actin was used as internal loading control. C) Quantitative analyses of 
889 NFATc1 and c-Fos protein expression relative to $\beta$-actin by densitometry were 890 conducted. D) MIF expression in BMMs treated with or without RANKL and CSB6B 891 was detected. E) Normalized MIF expression was quantified. F) MIF expression in 892 BMMs treated with or without Rankl, CSB6B and the proteasomal inhibitor MG132 or 893 protein synthesis Inhibitors Cycloheximide. G) Quantitative analyses of protein 894 expression of MIF relative to $\beta$-actin by densitometry were conducted. H) BMMs were 895 incubated with RANKL and CSB6B or untreated, and the immunoprecipitation with 896 MIF antibody was performed. The obtained immunoprecipitates were analyzed using 897 the indicated antibodies. I) TCPs from BMMs stimulated with RANKL without or with 898 CSB6B $(20 \mu \mathrm{M})$ for $0,10,20$, or $40 \mathrm{~min}$ (short time course) were extracted and 899 subjected to Western blot analysis. The protein expressions of p65, p-p65, IkB $\alpha, p-I k B \alpha$ 900 involved in early NF- $\mathrm{BB}$ signaling were assessed. Expression of $\beta$-actin was used as 901 internal loading control. J) Quantitative analyses of protein expression of p-IkBa, p$902 \mathrm{p} 65, \mathrm{p}-\mathrm{IKK} \alpha / \beta$ relative to $\beta$-actin by densitometry were conducted. K) $\mathrm{NF}-\kappa \mathrm{B}$ p65 903 nuclear translocation following CSB6B treatment was examined by 904 immunofluorescence microscopy. Cells were stained with specific antibody against p65 905 and nuclei were counterstained with DAPI. Scale bars, $25 \mu \mathrm{m}$. L) The percentage of 906 nuclear localized NF- $\mathrm{B}$ p 65 was quantified 
Fig.3
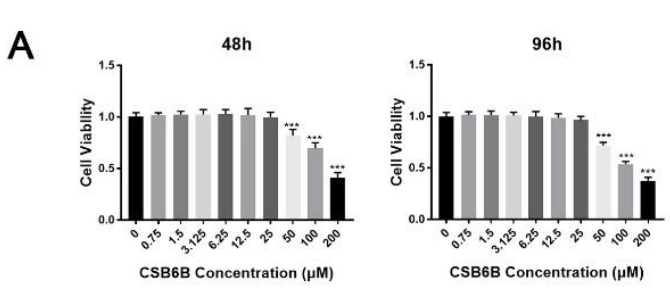

B

C

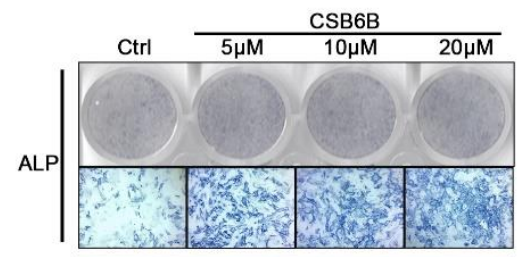

D
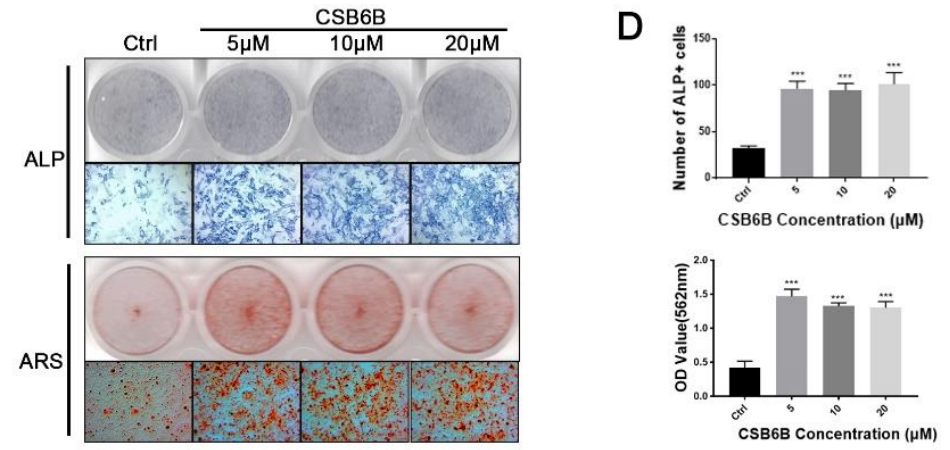

E
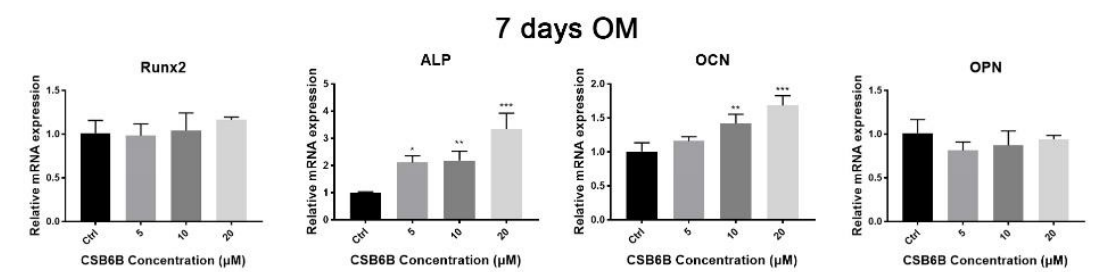

F 21 days $O M$
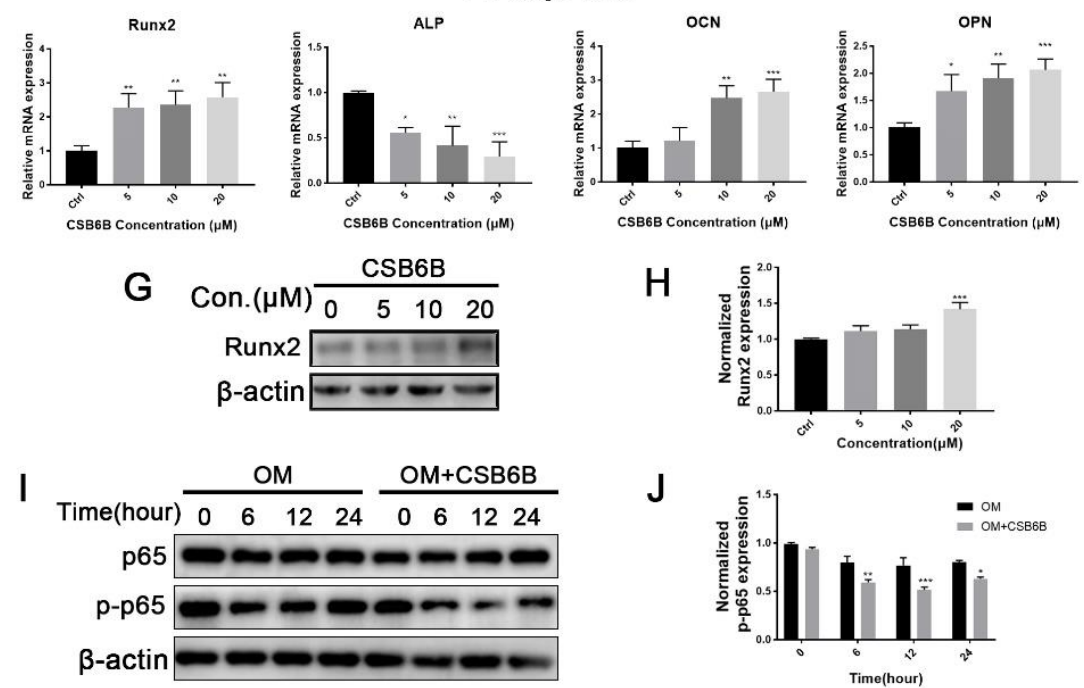

$\mathrm{H}$

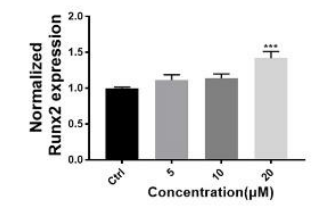

$\mathrm{J}$

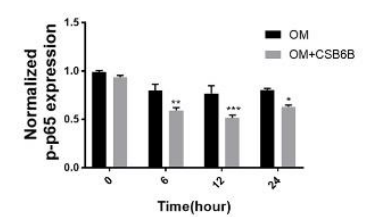

Figure 3

909 Fig.3 CSB6B potentiates osteoblast-mediated mineralization and bone nodule

910 formation. A) Through CCK-8 assay, cytotoxic effects of CSB6B on primary calvarial

911 osteoblasts were assessed at 48 and 96 h. B) Calculated IC50 value of CSB6B in 912 primary calvarial osteoblasts at $48 \mathrm{~h}$ was $61.93 \mu \mathrm{M}$. C) Primary calvarial osteoblasts 913 stimulated with osteogenic medium(OM) with or without CSB6B were cultured. After 
914 cells were cultured for 7d, ALP activity were stained and after cultured for $21 \mathrm{~d}$, 915 mineralized bone nodule formation with ARS were stained. D) The number of ALP916 positive cells and calcium deposits in bone nodules were quantified. E, F) The 917 osteogenesis related gene expression of Runx2, ALP, OCN, and OPN were analyzed by 918 qPCR using RNAs extracted from primary calvarial osteoblasts stimulated with 919 osteogenic media with or without indicated concentrations of CSB6B for 7 (E) or $21 \mathrm{~d}$ 920 (F), respectively. G) TCPs extracted from primary calvarial osteoblasts treated with 921 osteogenic media and CSB6B for 21d were immunoblotted using specific antibodies 922 against Runx2. Expression of $\beta$-actin was used as internal loading control. H) Runx2 923 protein expression at each concentration was normalized to $\beta$-actin expression. I) TCPs 924 extracted from primary calvarial osteoblasts treated with osteogenic media and CSB6B $925(20 \mu \mathrm{M})$ for $0,6,12$, or $24 \mathrm{~h}$ were subjected to Western blot analysis using specific 926 antibodies against p65 and p-p65. J) Quantitative analyses of p-p65 protein expression relative to $\beta$-actin by densitometry were conducted. 
Fig.4

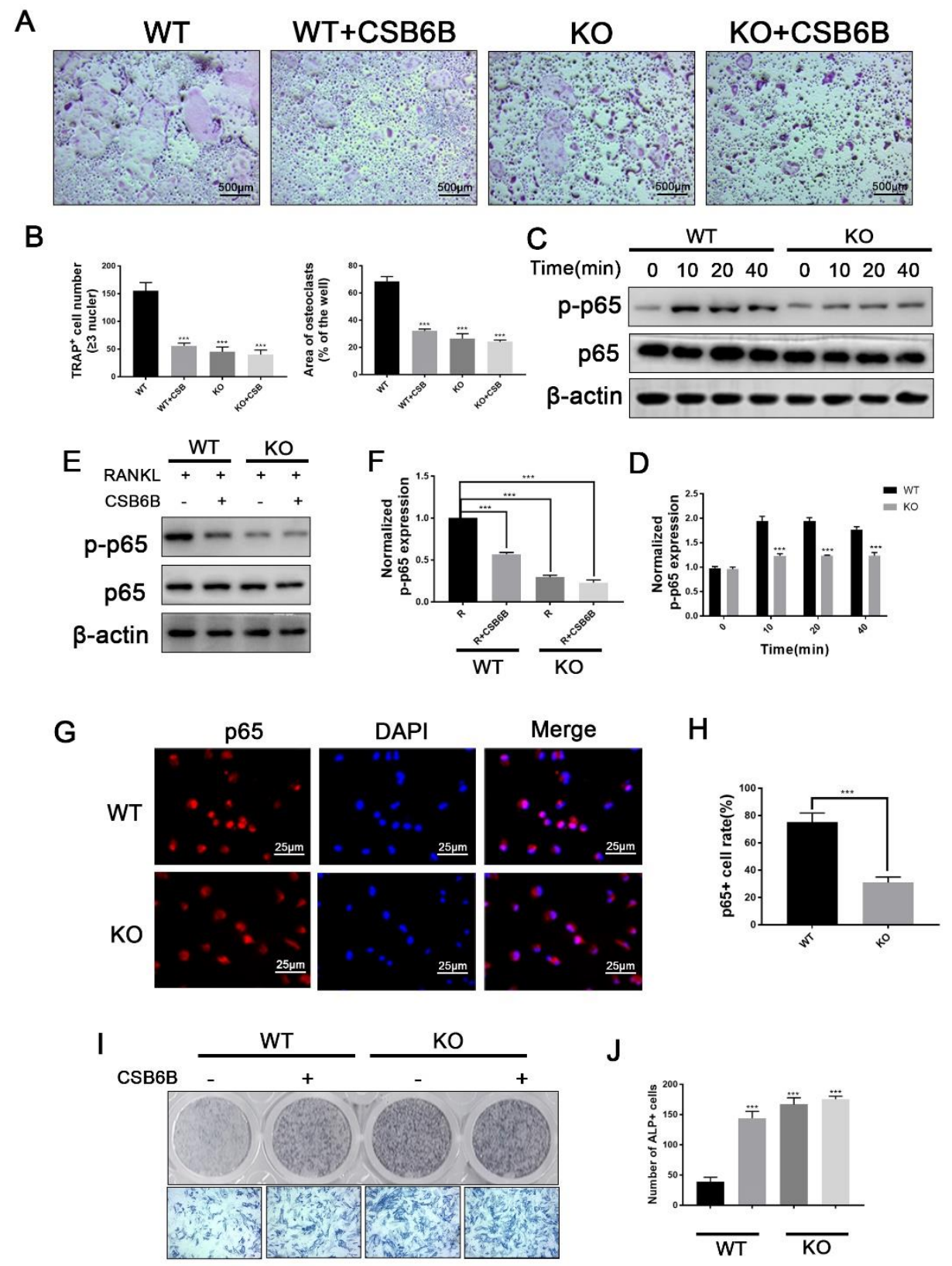

Figure 4

930 Fig.4 BMMs derived from MIF-KO mice exhibit lower osteoclast differentiation 931 potential as a result of impaired RANKL induced NF-kB signaling. A) BMMs 932 extracted from MIF KO and age-matched wild-type littermates were stimulated with 933 RANKL without or with $20 \mathrm{mM} \mathrm{CSB6B}$ for $5 \mathrm{~d}$ after which multinucleated osteoclasts 934 were fixed and stained for TRAP activity. Scale bars, $500 \mu \mathrm{m}$. B) The number and size 
935 (mean area) of TRAP-positive multinucleated osteoclasts $\geq 3$ nuclei were quantified. C)

936 TCPs from WT or KO BMMs stimulated with RANKL without or with $20 \mu \mathrm{M}$ CSB6B

937 for $0,10,20$, or $40 \mathrm{~min}$ (short time course) were extracted and subjected to Western

938 blot analysis. D) The protein expression of p-p65 were assessed. Expression of $\beta$-actin 939 was used as internal loading control. E) TCPs from WT or KO BMMs stimulated with

940 RANKL without or with $20 \mu \mathrm{M}$ CSB6B for 30mins were extracted and subjected to

941 Western blot analysis. F) Quantitative analyses of p-p65 protein expression relative to

$942 \beta$-actin by densitometry were conducted. G) NF-кB p65 nuclear translocation following

943 CSB6B treatment in WT and MIF-KO cells was examined by immunofluorescence

944 microscopy. Cells were stained with specific antibody against p65, and nuclei were

945 counterstained with DAPI. Scale bars, $25 \mu \mathrm{m}$. H) The percentage of nuclear NF- $\kappa B$ p65

946 was quantified. I) Primary calvarial osteoblasts of MIF KO and WT littermates were

947 stimulated with osteogenic media. After $7 \mathrm{~d}$ of culture, cells were stained for ALP

948 activity. J) The number of ALP-positive cells were quantified. 
Fig.5

A

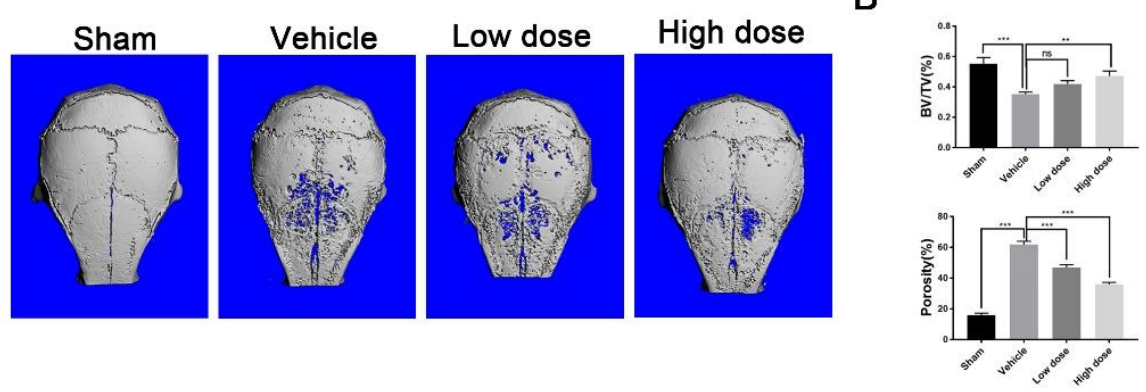

C

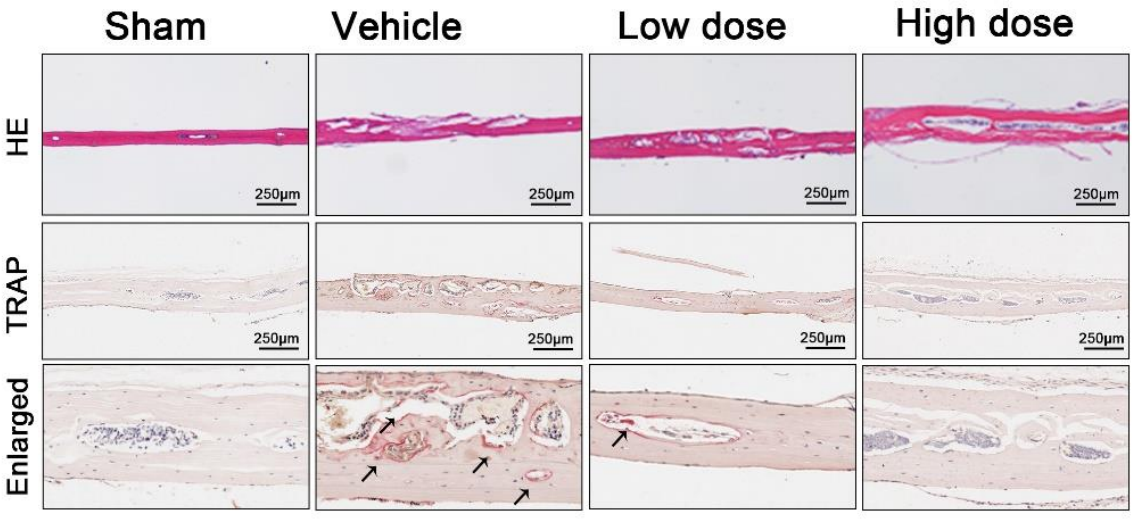

$\mathrm{E}$

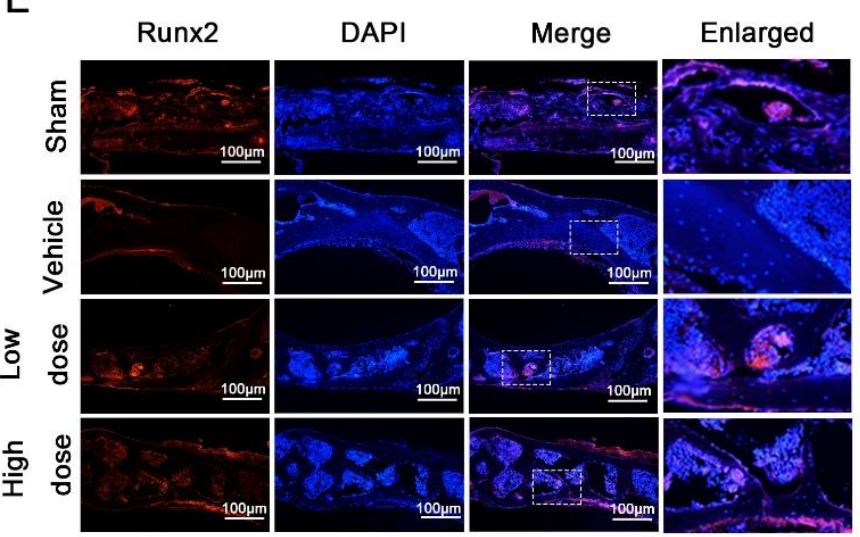

D

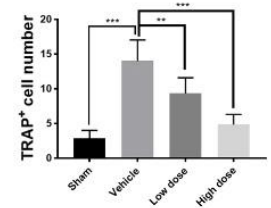

$\mathrm{F}$

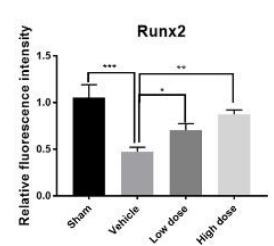

Figure 5

951 Fig.5 Administration of CSB6B protected against Ti particle-induced calvarial

952 osteolysis in vivo. A) micro CT reconstructions of whole calvarial tissue from sham-

953 treated, Ti particle-treated mice injected with PBS, Ti particle-treated mice injected

954 with $2 \mathrm{mg} / \mathrm{kg} \mathrm{CSB} 6 \mathrm{~B}$ (low dose), and Ti particle-treated mice injected with $8 \mathrm{mg} / \mathrm{kg}$

955 CSB6B (high dose). B) Bone morphometric parameters of the amount of bone 
956 resorption volume expressed as a percentage of porosity of the whole calvaria 957 (\%porosity) was measured. C) Histologic assessments of calvarial tissue sections by 958 hematoxylin-eosin (H\&E) and TRAP staining were carried out. Scale bars, $250 \mu \mathrm{m}$. D) 959 Histomorphometry analysis of the total number of TRAP-positive osteoclasts ( $>5$ 960 nuclei). E) Immunofluorescence analysis of the expression of Runx2 in calvarial tissue 961 sections from each experimental mouse group. Sections were counterstained with DAPI. 962 Scale bars, $100 \mu \mathrm{m}$. F) Quantitative analysis of the relative fluorescence intensity of 963 Runx 2 in each experimental group. Images presented are representative of $\geq 3$ sections 964 for each group, and data are expressed as means \pm SD. $* \mathrm{P} \leq 0.05, * * \mathrm{P} \leq 0.01, * * * \mathrm{P} \leq 0.005$ 965 when compared with vehicle control. 


\section{Fig.6}

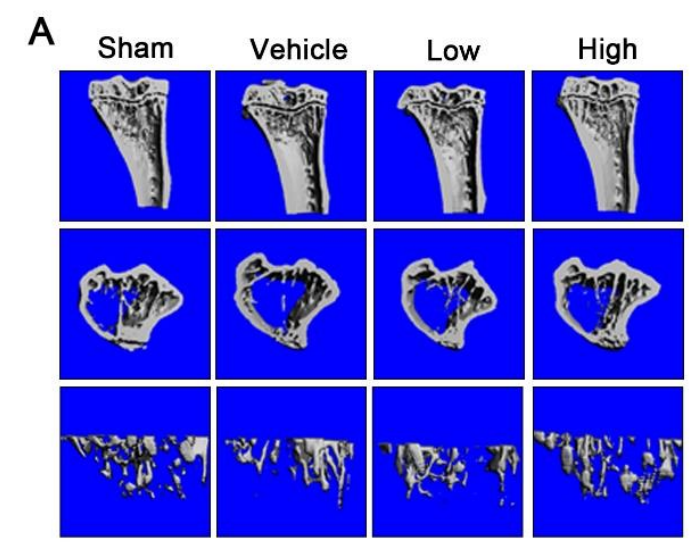

B
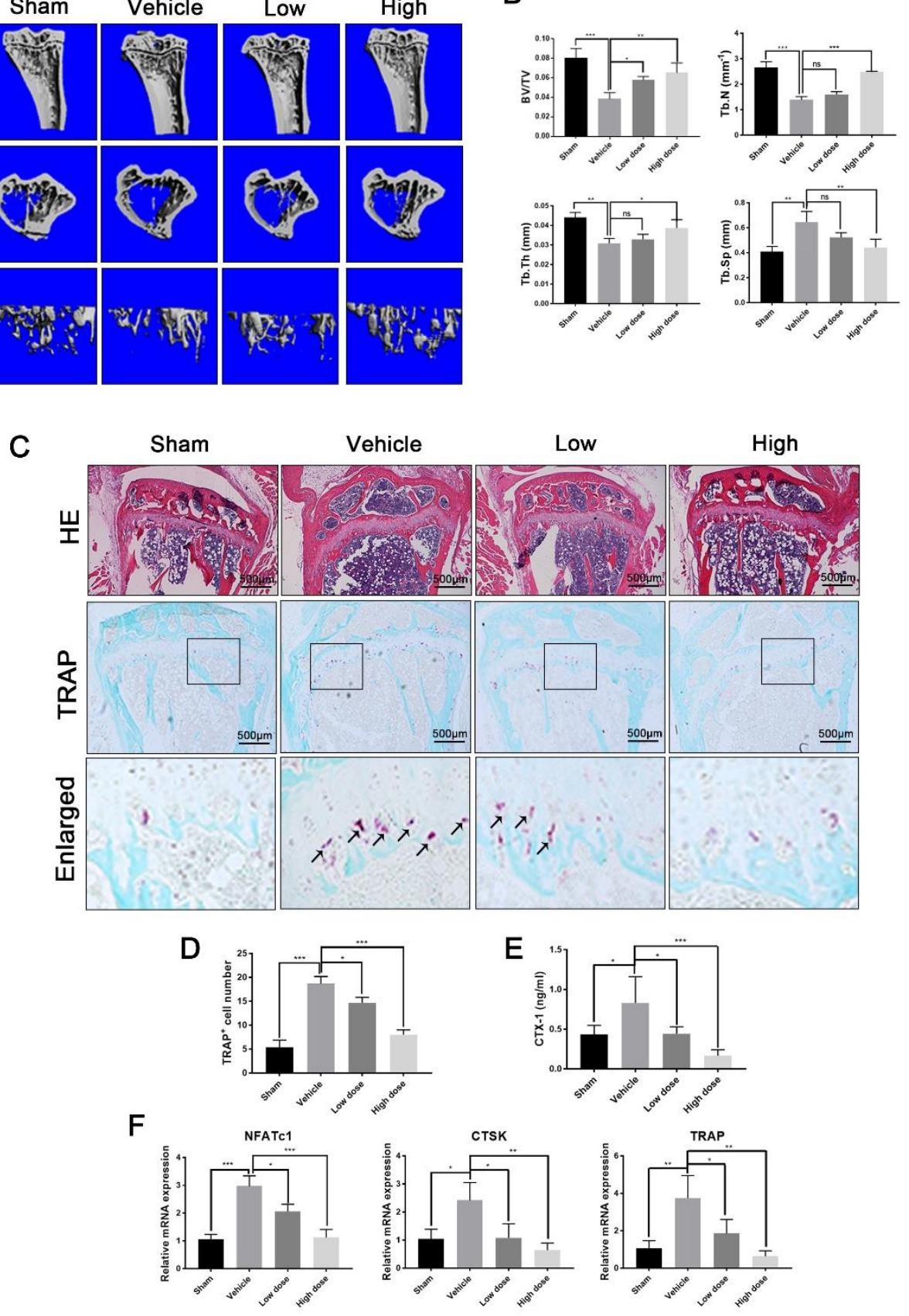

Figure 6

Fig.6. Administration of CSB6B alleviated OVX-induced osteoporosis in vivo. A)

969 The $\mu \mathrm{CT}$ scaning of the tibial bone from sham-treated, OVX injected with PBS

970 (vehicle), OVX injected with $2 \mathrm{mg} / \mathrm{kg}$ CSB6B (low dose), and OVX injected with 8

$971 \mathrm{mg} / \mathrm{kg}$ CSB6B (high dose). B) Bone microstructural parameters analyzed include

972 percentage BV/TV (\%), Tb.N, Tb.Th, and Tb.Sp. C) Histologic assessment of tibial 
973 bone sections by H\&E and TRAP staining of four groups. Scale bars, $500 \mu \mathrm{m}$. D) The 974 total number of TRAP-positive osteoclasts by TRAP staining were determined . E) To 975 assess the relative levels of osteoclastic bone resorptive activity in each experimental 976 mouse group, serum concentrations of CTX-1 $(n=6)$ were measured by ELISA. F) The 977 gene expression of NFATc1, CTSK, and TRAP were analyzed by qPCR using RNAs 978 extracted from bone tissues from each experimental mouse group. N.s., not significant. 979 Images presented are representative of $\geq 3$ sections for each group, and data are 980 expressed as means $\pm \mathrm{SD}$. $* \mathrm{P} \leq 0.05, * * \mathrm{P} \leq 0.01, * * * \mathrm{P} \leq 0.005$ when compared with vehicle 981 control. 
Fig.7

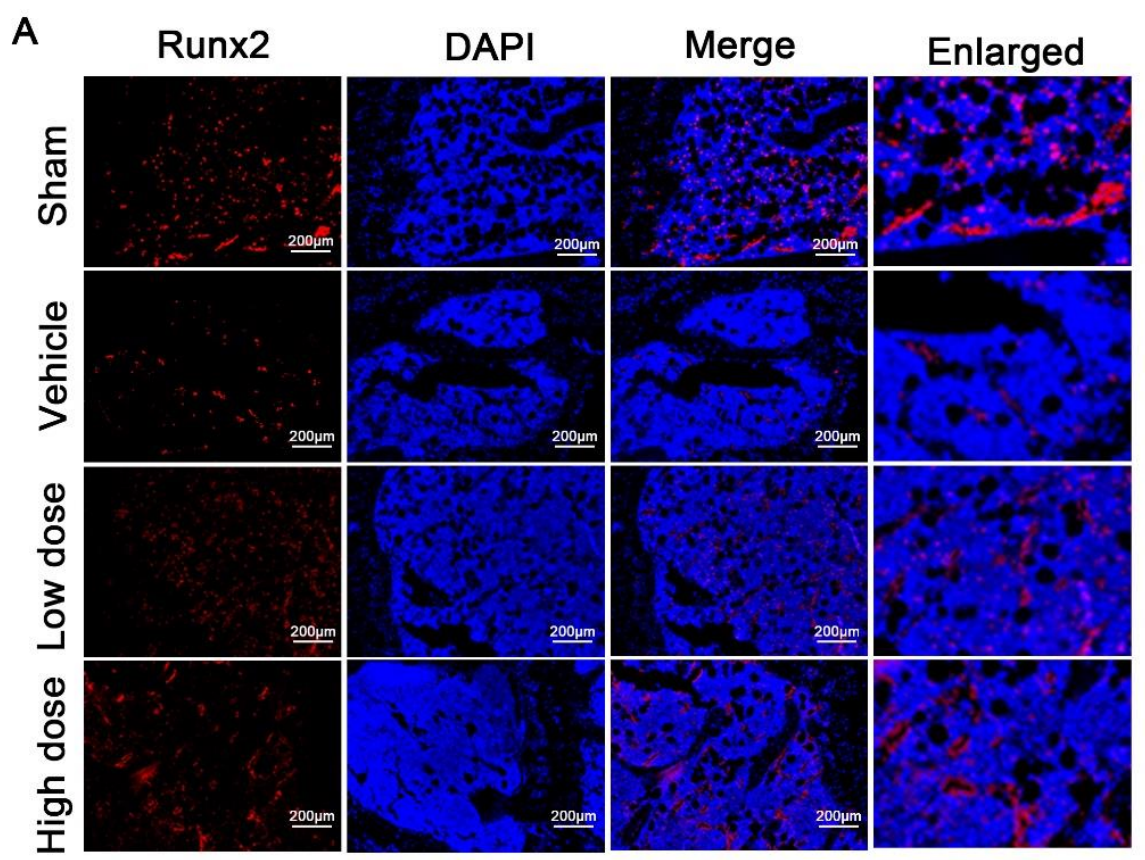

B

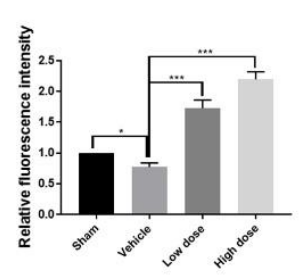

D
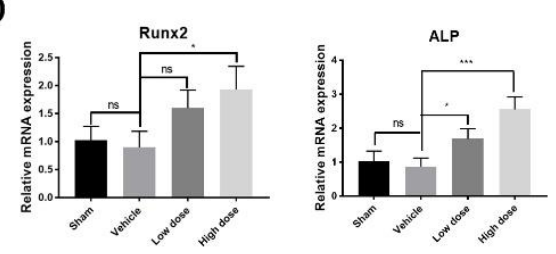

C

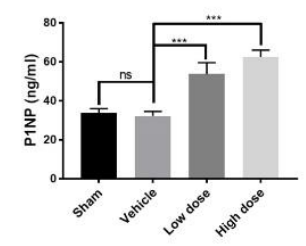

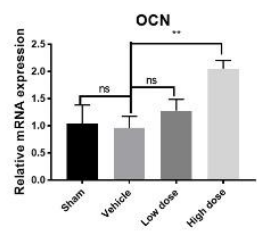

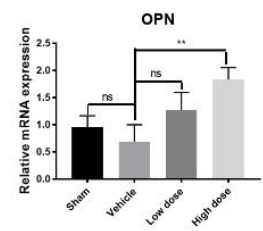

Figure 7

Fig.7 Administration of CSB6B promoted osteoblast activity in OVX-induced osteoporosis. A) Immunofluorescence analysis of the expression of Runx2 in tibial

986 tissue sections from each experimental mouse group. Sections were counterstained with 987 DAPI. Scale bars, $200 \mu \mathrm{m}$. B) The relative fluorescence intensity of Runx2 was 988 quantified. C) To assess the relative levels of osteoblastic bone formation activity in 
989 each experimental mouse group, serum concentrations of P1NP $(n=6)$ were measured 990 by ELISA. D) Osteoblast marker genes such as Runx2, ALP, OCN, and OPN were 991 analyzed by qPCR using RNAs extracted from bone tissues from each experimental 992 mouse group. N.s., not significant. Images presented are representative of $\geq 3$ sections 993 for each group, and data are expressed as means \pm SD. $* \mathrm{P} \leq 0.05, * * \mathrm{P} \leq 0.01, * * * \mathrm{P} \leq 0.005$ 994 when compared with vehicle control. 
A

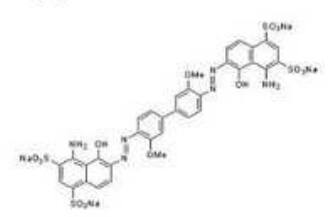

D
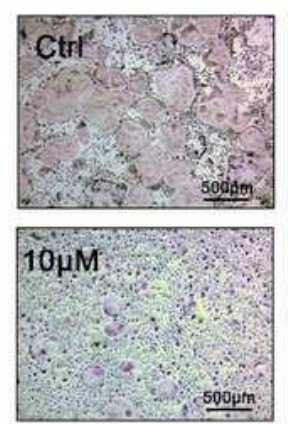

F

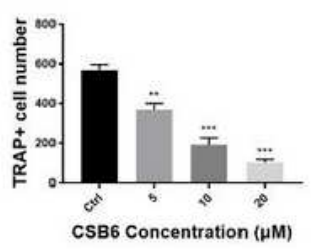

H
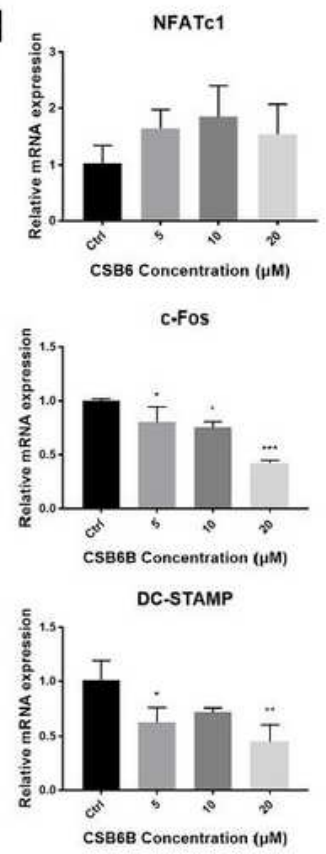

B
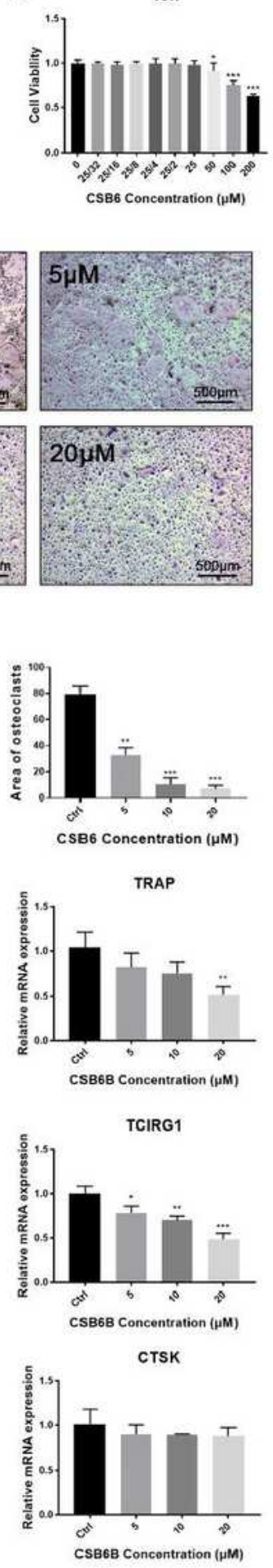

96h

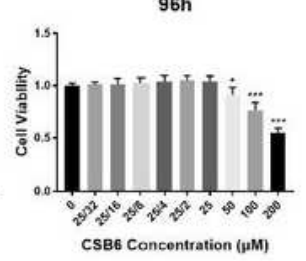

C

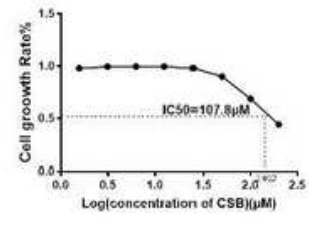

E
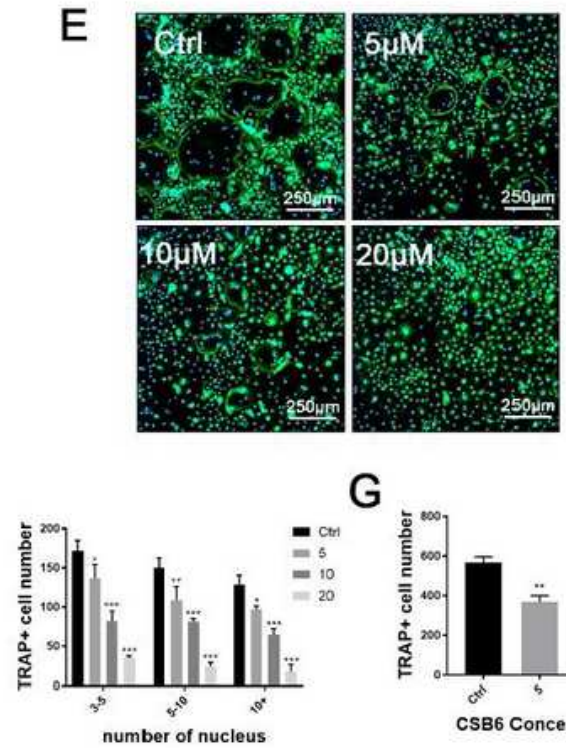

G

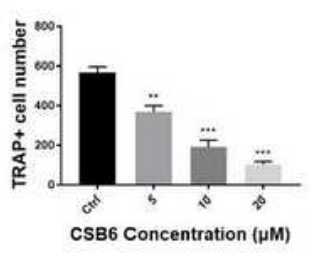

I
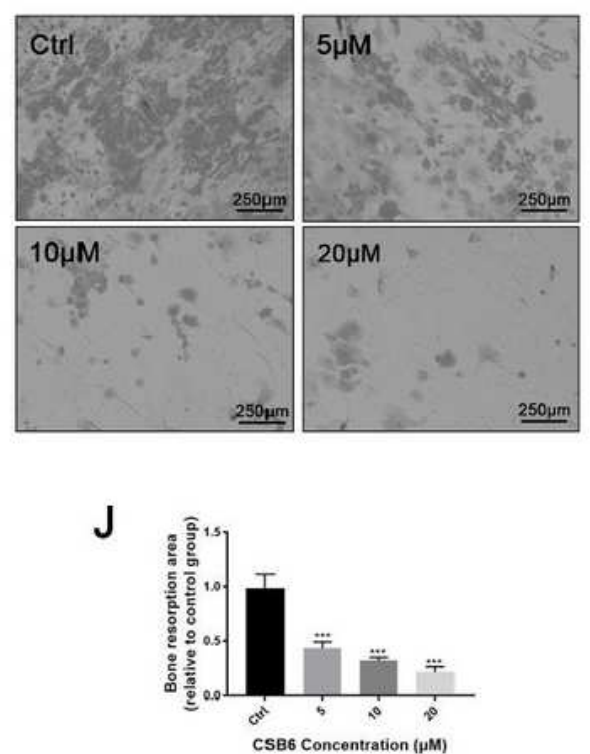

Figure 1

CSB6B inhibited osteoclast formation in a dose-dependent in vitro. A) The structure of CSB6B. B) Cytotoxic effects of CSB6B on BMMs were assessed by CCK-8 cell viability/cytoxicity assay at 48 and 96 h. C) IC50 values obtained for the activity of CSB6B against BMMs. D) BMMs were treated with 
RANKL(50 ng/ml), M-CSF(40 ng/ml) without or with different concentrations (Con.) of CSB6B for $5 \mathrm{~d}$, after that osteoclasts were fixed and stained for TRAP. Scale bars, $500 \mu \mathrm{m}$. F) The number and size (mean area) of TRAP-positive multinucleated osteoclasts with 5 nuclei were quantified. The distribution of TRAP-positive osteoclasts with 3-5, 5-10, or 10 nuclei were also determined. E) Following treatment with indicated concentrations of CSB6B, actin belts were stained with rhodamine-conjugated phalloidin and analyzed by immunofluorescence microscopy. Nuclei were counterstained with DAPI. Scale bars, 250 $\mu \mathrm{m} . \mathrm{G})$ The number of actin belts in four groups were quantified. $H$ ) Expression of osteoclasts genes in BMMs simulated with RANKL and different concentration of CSB6B for $5 \mathrm{~d}$ such as NFATc1, c-Fos, DCSTAMP, TRAP, TCIRG1 and CTSK were analyzed by qPCR. I) Bone resorptive activity of BMMs cultured on bovine bone discs were assessed by scanning electron microscopy following treatment with CSB6B $(20 \mu \mathrm{M})$. Scale bars, $250 \mu \mathrm{m}$. J) The mean resorption pit area was quantified. Images presented are representative of $\geq 3$ independent experiments, and data are expressed as means $\pm S D$. ${ }^{*} P \leq 0.05,{ }^{*} P \leq 0.01$, $\star * \star P \leq 0.005$ when compared with control group. 

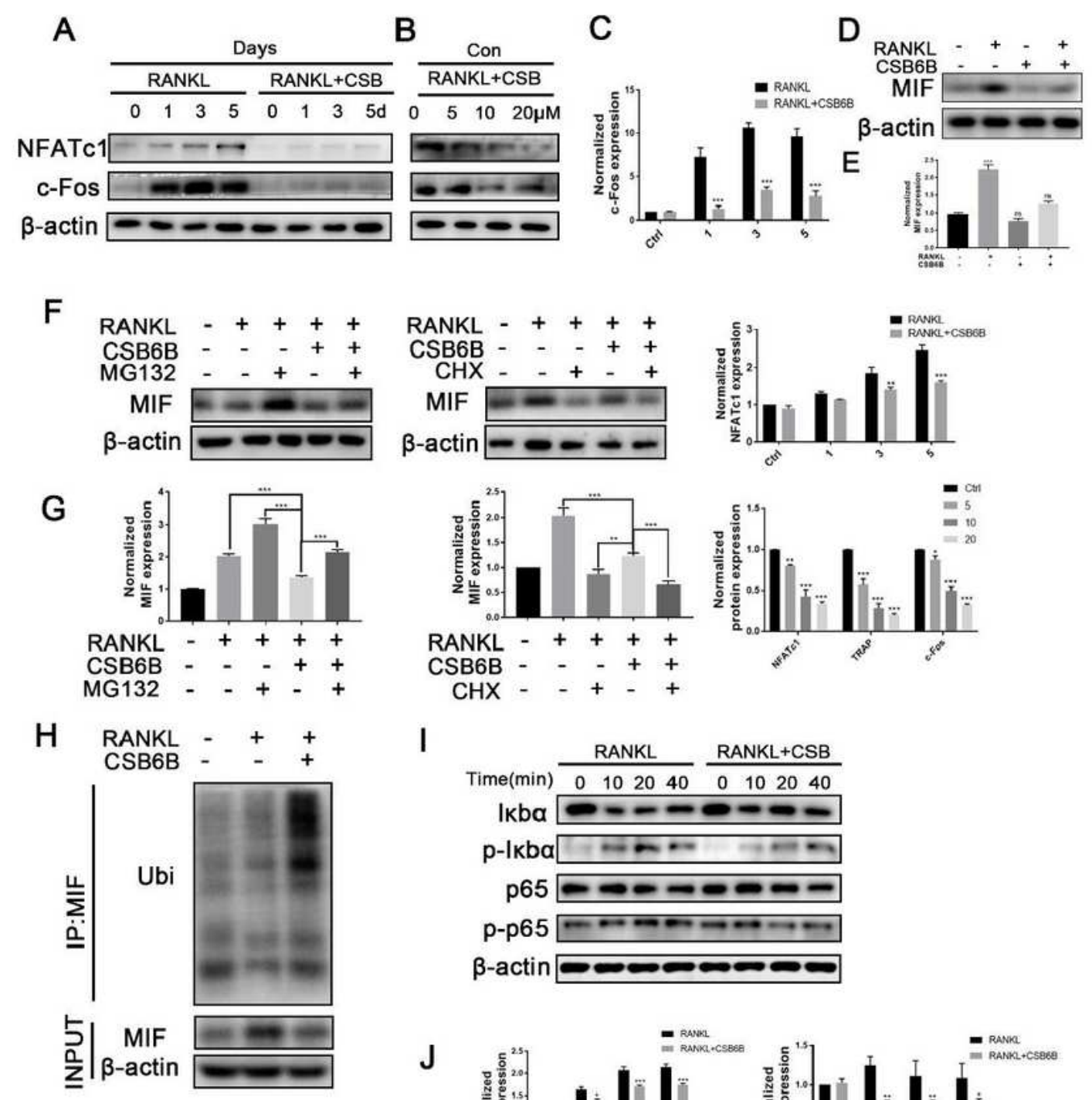

I

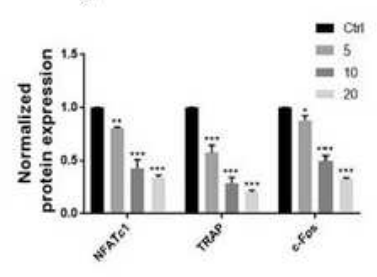

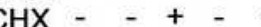

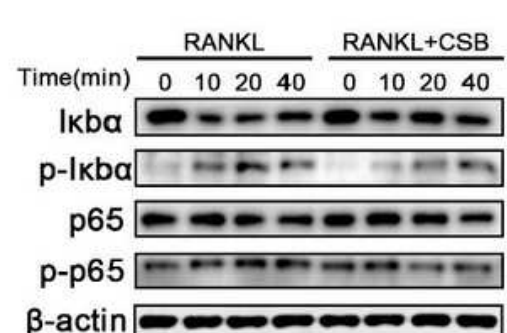

$\beta$-actin

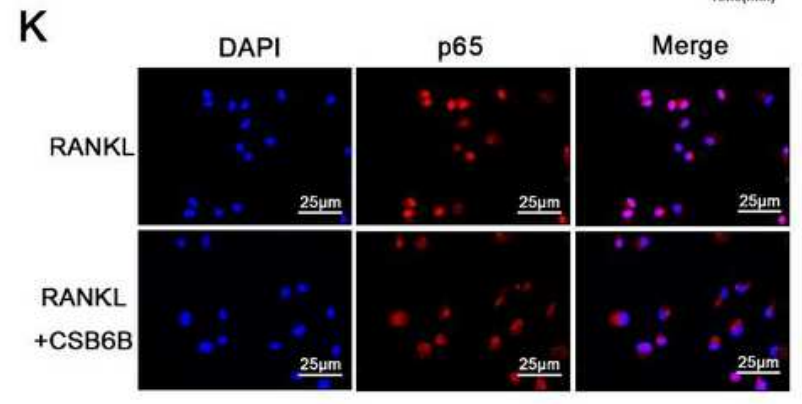

L
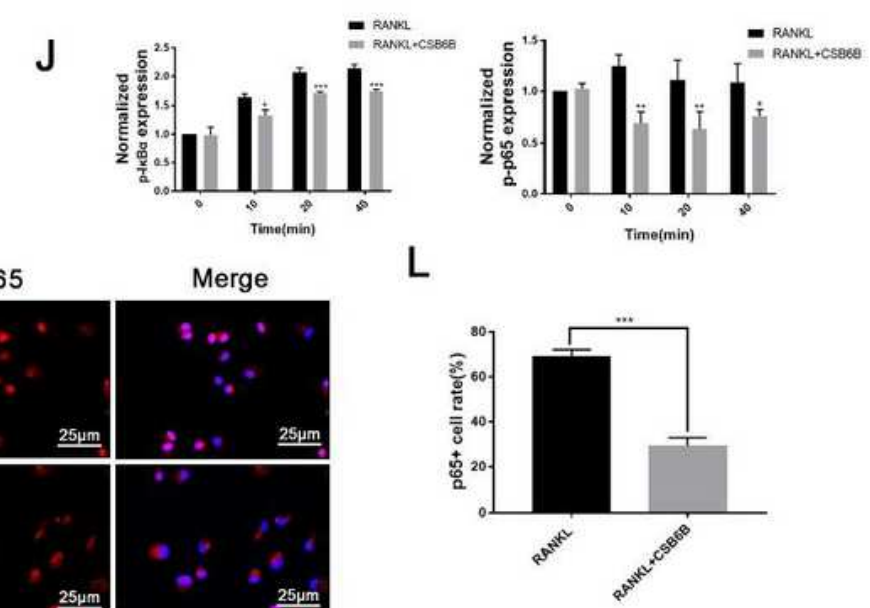

Figure 2

CSB6B inhibits early NF-KB signaling. A, B) TCPs from BMMs stimulated with RANKL without or with CSB6B $(20 \mu \mathrm{M})$ for $0,1,3$, or $5 \mathrm{~d}$ (late time course) or with indicated concentrations of CSB6B (dose dependent) for $5 \mathrm{~d}$ were extracted and subjected to Western blot analysis with specific antibodies against NFATc 1 and c-Fos. Expression of $\beta$-actin was used as internal loading control. C) Quantitative analyses of NFATc1 and c-Fos protein expression relative to $\beta$-actin by densitometry were conducted. D) MIF 
expression in BMMs treated with or without RANKL and CSB6B was detected. E) Normalized MIF expression was quantified. F) MIF expression in BMMs treated with or without Rankl, CSB6B and the proteasomal inhibitor MG132 or protein synthesis Inhibitors Cycloheximide. G) Quantitative analyses of protein expression of MIF relative to $\beta$-actin by densitometry were conducted. $H$ ) BMMs were incubated with RANKL and CSB6B or untreated, and the immunoprecipitation with MIF antibody was performed. The obtained immunoprecipitates were analyzed using the indicated antibodies. I) TCPs from BMMs stimulated with RANKL without or with CSB6B $(20 \mu \mathrm{M})$ for $0,10,20$, or 40 min (short time course) were extracted and subjected to Western blot analysis. The protein expressions of p65, p-p65, lkBa, p-lkBa involved in early NF-KB signaling were assessed. Expression of $\beta$-actin was used as internal loading control. J) Quantitative analyses of protein expression of $p-1 k B a, p-p 65, p-1 K K a / \beta$ relative to $\beta$-actin by densitometry were conducted. K) NF-KB p65 nuclear translocation following CSB6B treatment was examined by immunofluorescence microscopy. Cells were stained with specific antibody against p65 and nuclei were counterstained with DAPI. Scale bars, $25 \mu \mathrm{m}$. L) The percentage of nuclear localized NF-KB p65 was quantified 
A
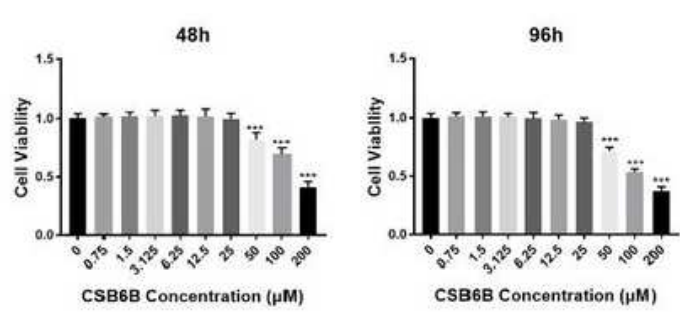

B

C

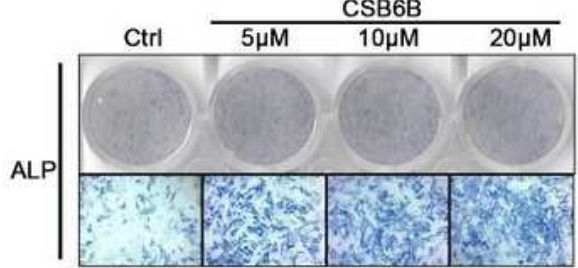

D
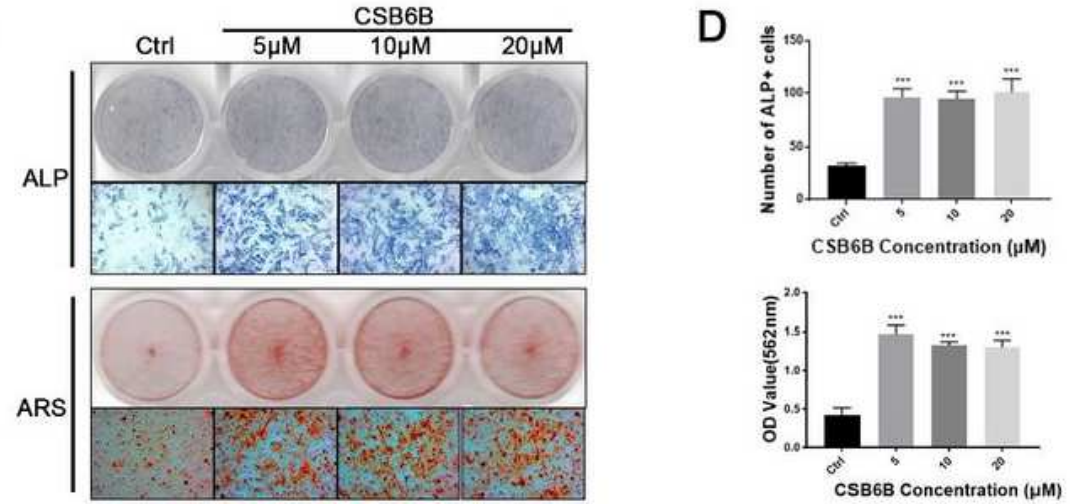

E

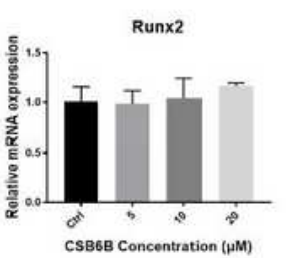

$\mathbf{F}$
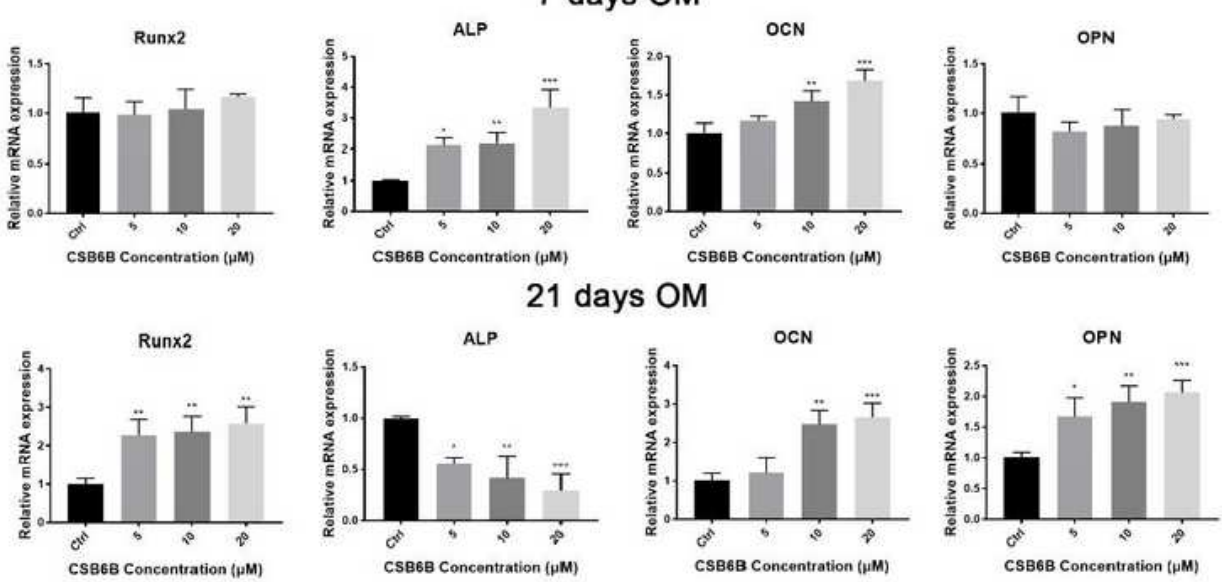

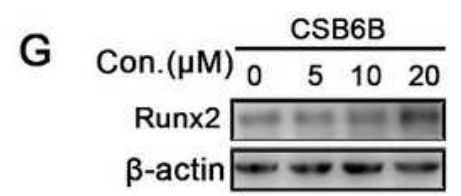

$\mathrm{H}$
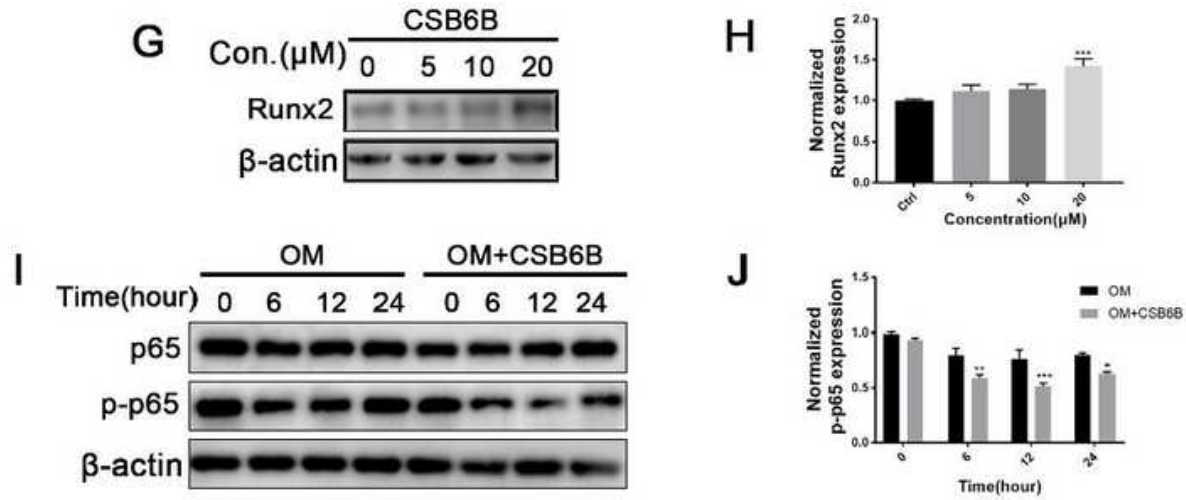

\section{Figure 3}

CSB6B potentiates osteoblast-mediated mineralization and bone nodule formation. A) Through CCK-8 assay, cytotoxic effects of CSB6B on primary calvarial osteoblasts were assessed at 48 and $96 \mathrm{~h}$. B) Calculated IC50 value of CSB6B in primary calvarial osteoblasts at $48 \mathrm{~h}$ was $61.93 \mu \mathrm{M}$. C) Primary calvarial osteoblasts stimulated with osteogenic medium(OM) with or without CSB6B were cultured. After cells were cultured for 7d, ALP activity were stained and after cultured for $21 \mathrm{~d}$, mineralized bone nodule 
formation with ARS were stained. D) The number of ALP-positive cells and calcium deposits in bone nodules were quantified. E, F) The osteogenesis related gene expression of Runx2, ALP, OCN, and OPN were analyzed by qPCR using RNAs extracted from primary calvarial osteoblasts stimulated with osteogenic media with or without indicated concentrations of CSB6B for 7 (E) or $21 d(F)$, respectively. $G$ ) TCPs extracted from primary calvarial osteoblasts treated with osteogenic media and CSB6B for 21d were immunoblotted using specific antibodies against Runx2. Expression of $\beta$-actin was used as internal loading control. H) Runx2 protein expression at each concentration was normalized to $\beta$-actin expression. I) TCPs extracted from primary calvarial osteoblasts treated with osteogenic media and CSB6B $(20 \mu \mathrm{M})$ for $0,6,12$, or $24 \mathrm{~h}$ were subjected to Western blot analysis using specific antibodies against $\mathrm{p} 65$ and $\mathrm{p}$ p65. J) Quantitative analyses of $p$-p65 protein expression relative to $\beta$-actin by densitometry were conducted. 
A

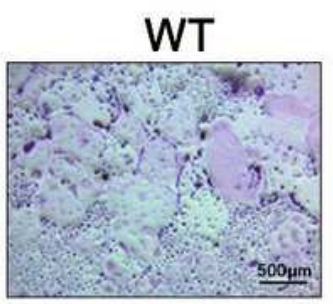

WT+CSB6B
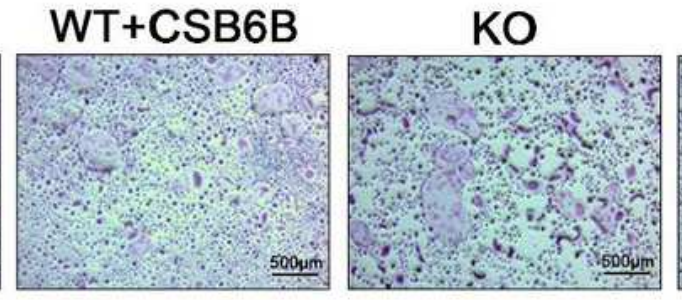

$\mathrm{KO}+\mathrm{CSB} 6 \mathrm{~B}$

B
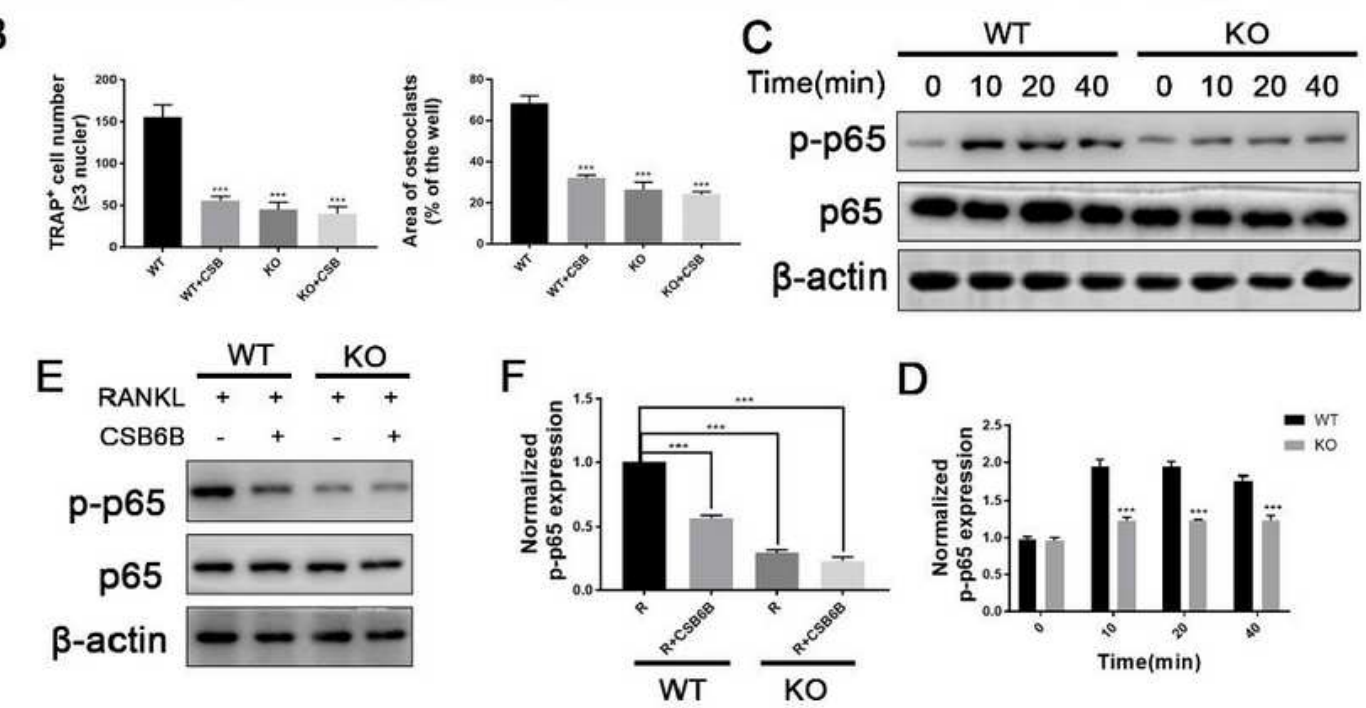

D
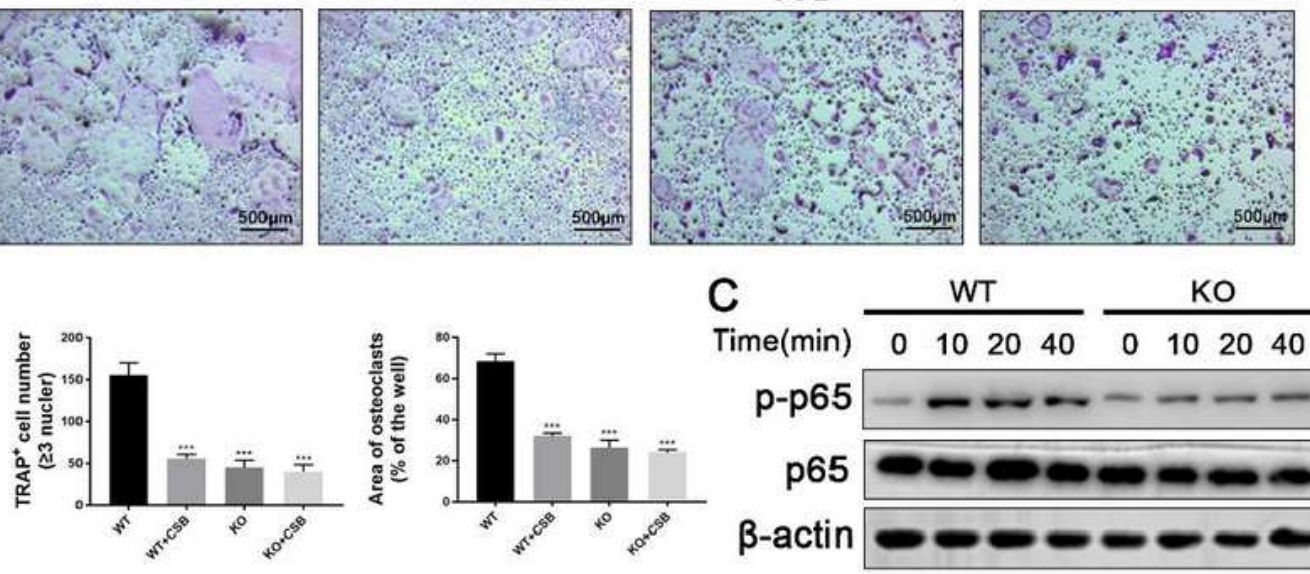

G
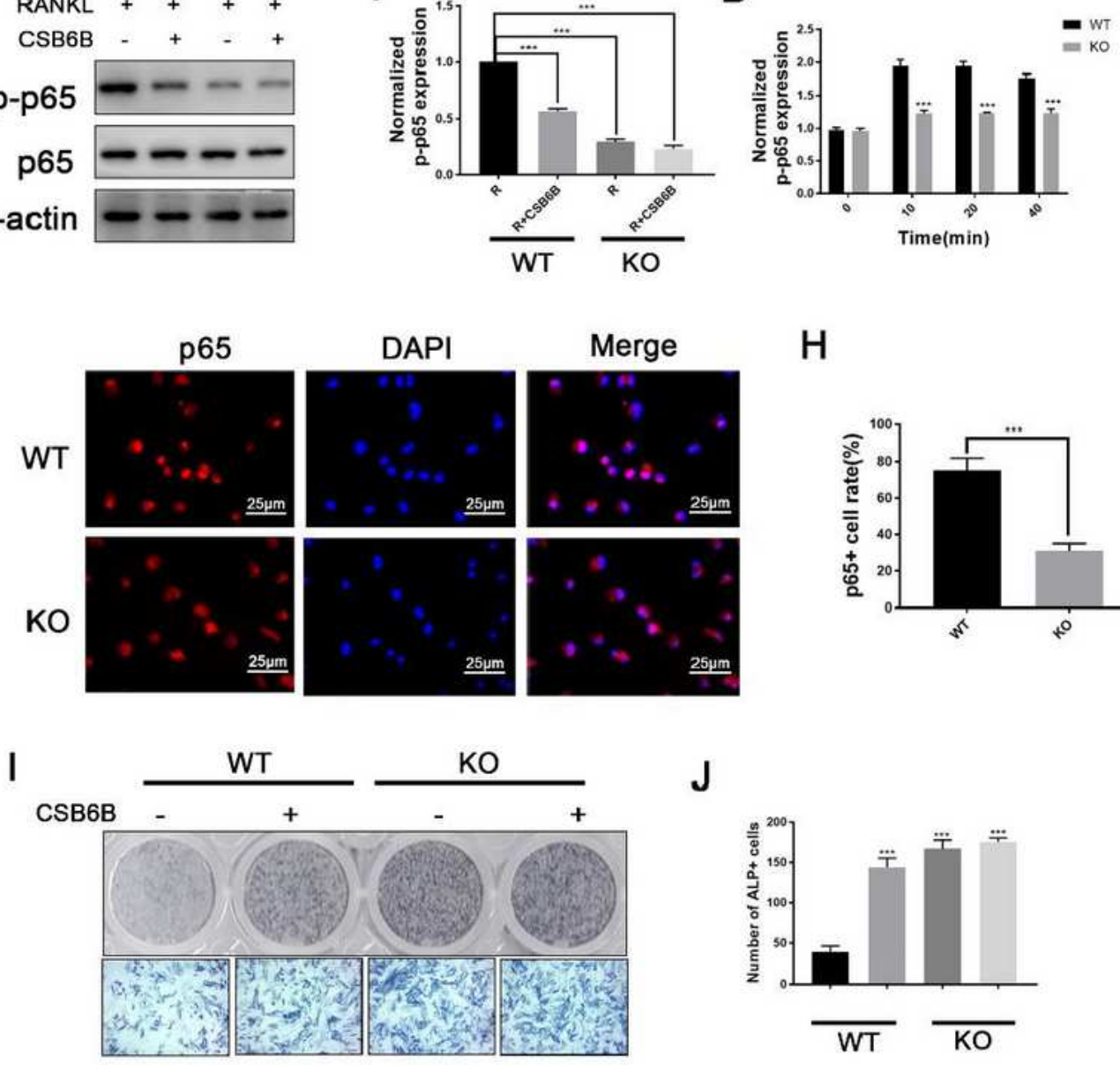

\section{Figure 4}

BMMs derived from MIF-KO mice exhibit lower osteoclast differentiation potential as a result of impaired RANKL induced NF-KB signaling. A) BMMs extracted from MIF KO and age-matched wild-type littermates were stimulated with RANKL without or with $20 \mathrm{mM}$ CSB6B for $5 \mathrm{~d}$ after which multinucleated osteoclasts were fixed and stained for TRAP activity. Scale bars, $500 \mu \mathrm{m}$. B) The number and size (mean area) of TRAP-positive multinucleated osteoclasts $\geq 3$ nuclei were quantified. C) TCPs from WT or KO 
BMMs stimulated with RANKL without or with $20 \mu \mathrm{M}$ CSB6B for $0,10,20$, or 40 min (short time course) were extracted and subjected to Western blot analysis. D) The protein expression of p-p65 were assessed. Expression of $\beta$-actin was used as internal loading control. E) TCPs from WT or KO BMMs stimulated with RANKL without or with $20 \mu \mathrm{M}$ CSB6B for 30 mins were extracted and subjected to Western blot analysis. F) Quantitative analyses of p-p65 protein expression relative to $\beta$-actin by densitometry were conducted. G) NF-KB p65 nuclear translocation following CSB6B treatment in WT and MIF-KO cells was examined by immunofluorescence microscopy. Cells were stained with specific antibody against p65, and nuclei were counterstained with DAPI. Scale bars, $25 \mu \mathrm{m}$. H) The percentage of nuclear NF-kB p65 was quantified. I) Primary calvarial osteoblasts of MIF KO and WT littermates were stimulated with osteogenic media. After $7 \mathrm{~d}$ of culture, cells were stained for ALP activity. J) The number of ALP-positive cells were quantified. 
A
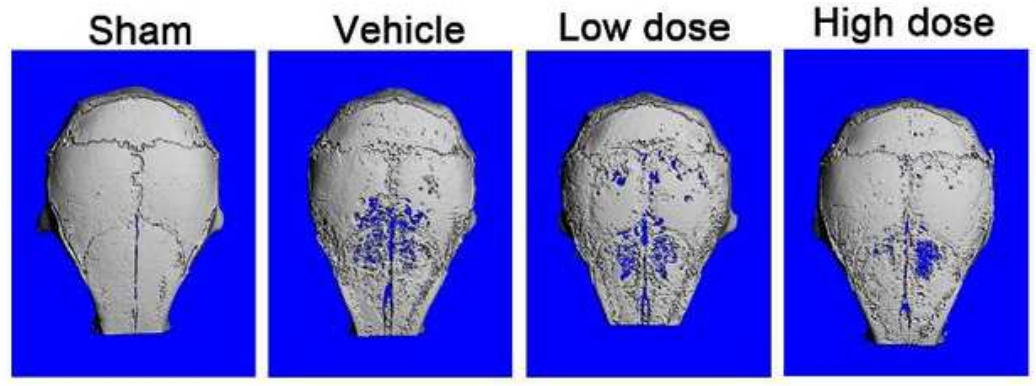

B
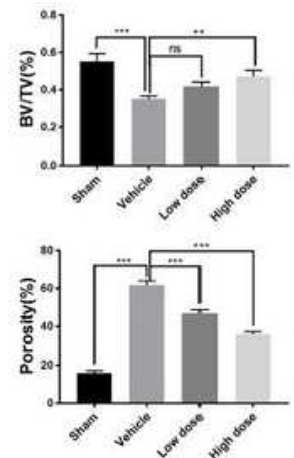

C

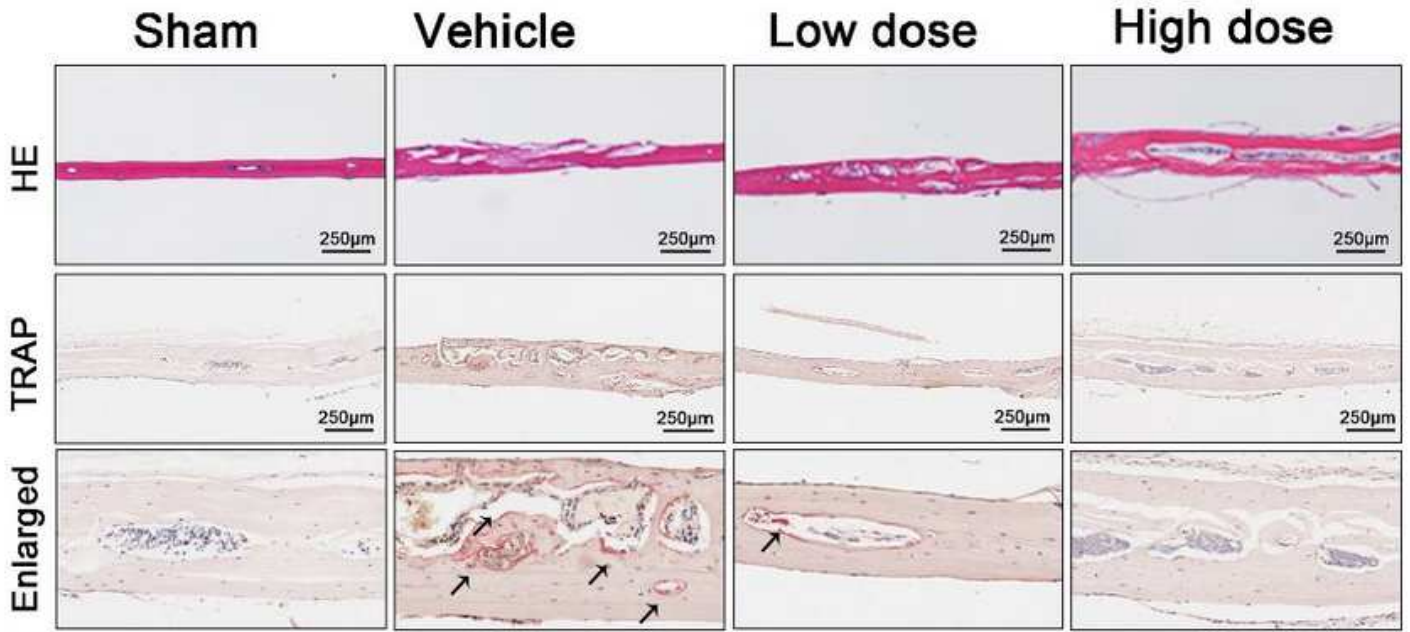

E
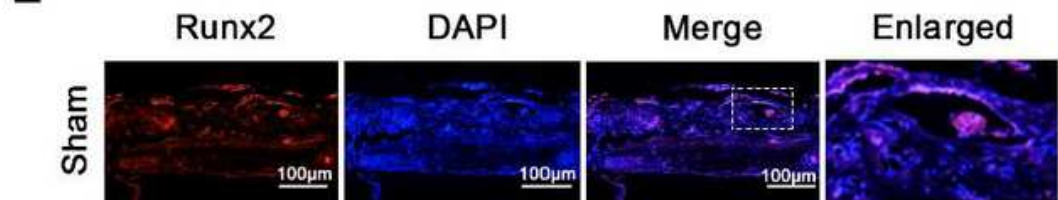

D
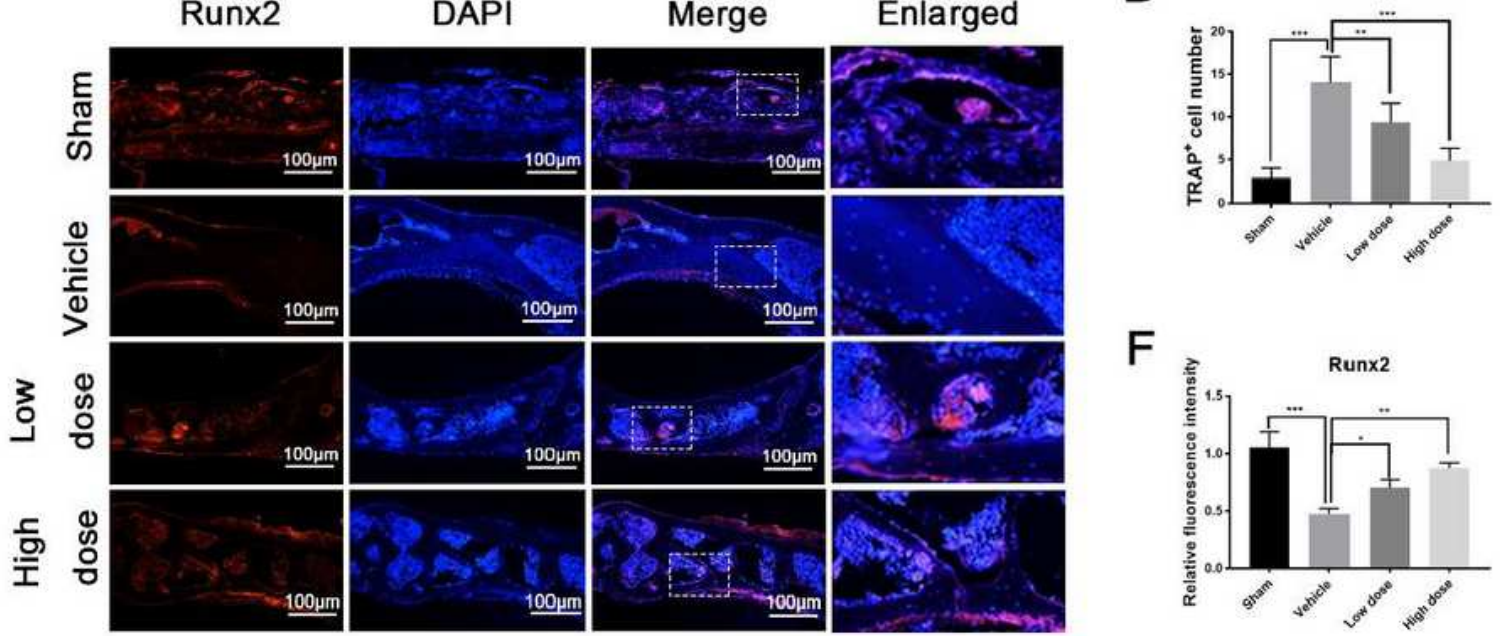

\section{Figure 5}

Administration of CSB6B protected against Ti particle-induced calvarial osteolysis in vivo. A) micro CT reconstructions of whole calvarial tissue from sham-treated, Ti particle-treated mice injected with PBS, Ti particle-treated mice injected with $2 \mathrm{mg} / \mathrm{kg}$ CSB6B (low dose), and Ti particle-treated mice injected with $8 \mathrm{mg} / \mathrm{kg}$ CSB6B (high dose). B) Bone morphometric parameters of the amount of bone resorption volume expressed as a percentage of porosity of the whole calvaria (\%porosity) was measured. C) Histologic 
assessments of calvarial tissue sections by hematoxylin-eosin (H\&E) and TRAP staining were carried out. Scale bars, $250 \mu \mathrm{m}$. D) Histomorphometry analysis of the total number of TRAP-positive osteoclasts ( $\mathbb{\square} \mathbf{5}$ nuclei). E) Immunofluorescence analysis of the expression of Runx2 in calvarial tissue sections from each experimental mouse group. Sections were counterstained with DAPI. Scale bars, $100 \mu \mathrm{m}$. F) Quantitative analysis of the relative fluorescence intensity of Runx2 in each experimental group. Images presented are representative of $\geq 3$ sections for each group, and data are expressed as means \pm SD. ${ }^{*} \mathrm{P} \leq 0.05, * * \mathrm{P} \leq 0.01,{ }^{*} * \mathrm{P} \leq 0.005$ when compared with vehicle control.
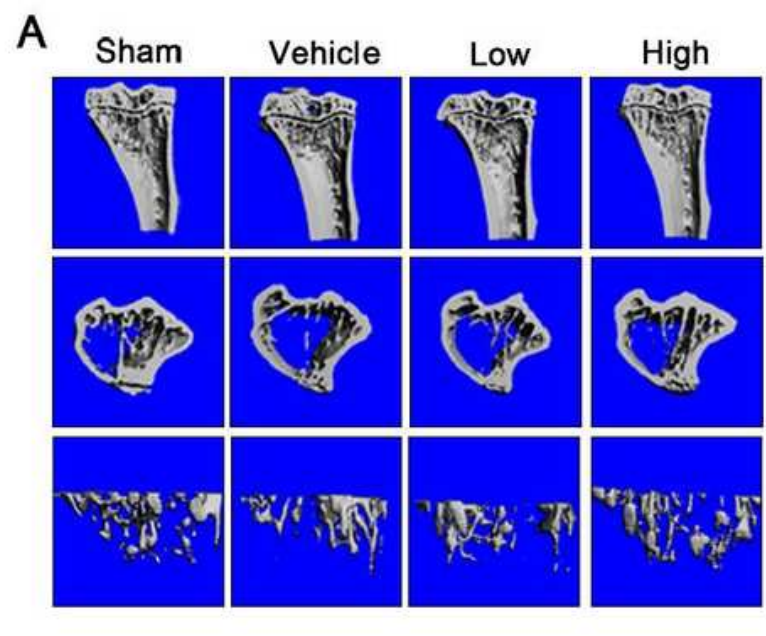

B
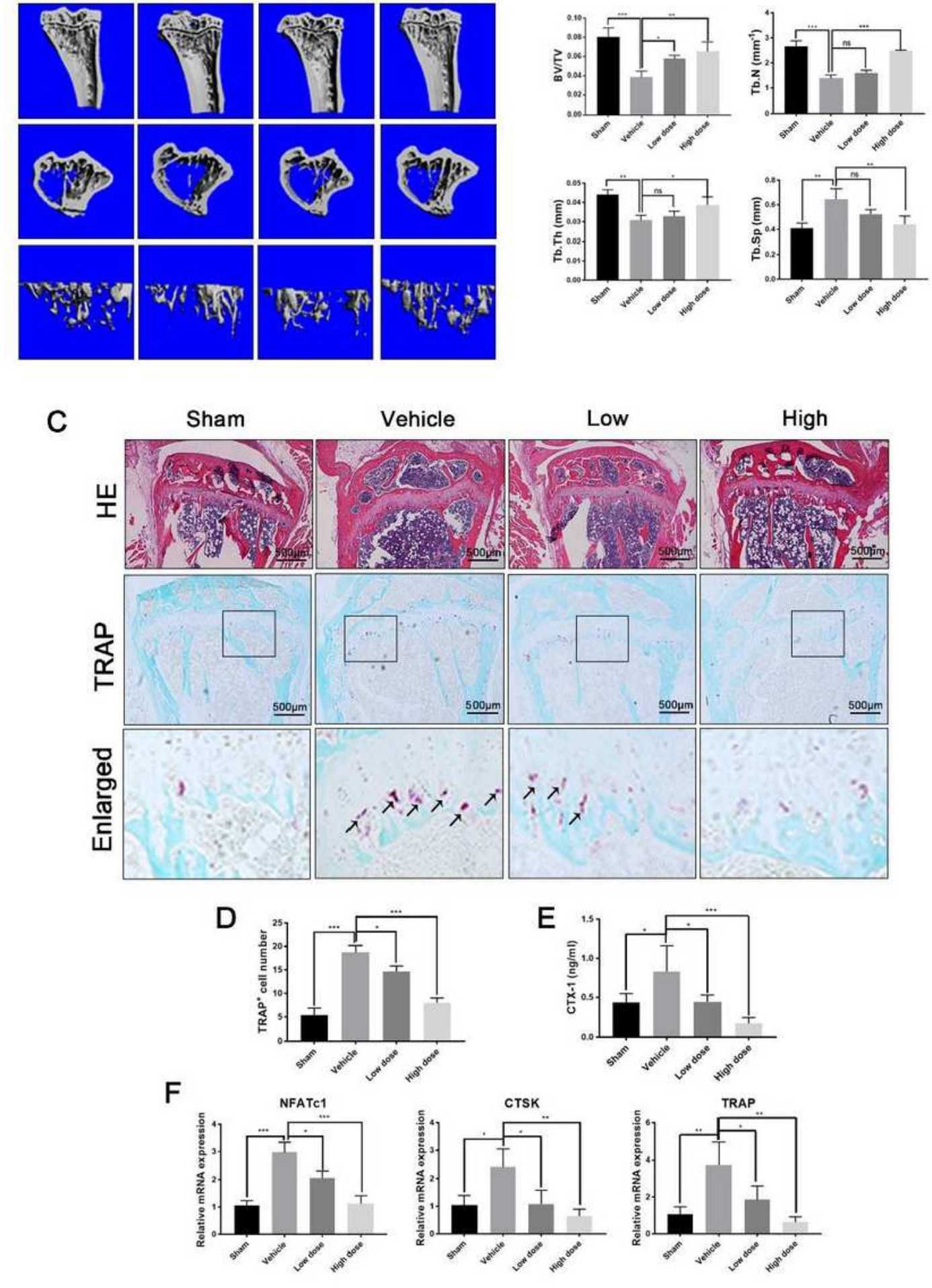


\section{Figure 6}

Administration of CSB6B alleviated OVX-induced osteoporosis in vivo. A) The $\mu \mathrm{CT}$ scaning of the tibial bone from sham-treated, OVX injected with PBS (vehicle), OVX injected with $2 \mathrm{mg} / \mathrm{kg}$ CSB6B (low dose), and OVX injected with $8 \mathrm{mg} / \mathrm{kg}$ CSB6B (high dose). B) Bone microstructural parameters analyzed include percentage BV/TV (\%), Tb.N, Tb.Th, and Tb.Sp. C) Histologic assessment of tibial bone sections by H\&E and TRAP staining of four groups. Scale bars, $500 \mu \mathrm{m}$. D) The total number of TRAP-positive osteoclasts by TRAP staining were determined. E) To assess the relative levels of osteoclastic bone resorptive activity in each experimental mouse group, serum concentrations of CTX-1 $(n=6)$ were measured by ELISA. F) The gene expression of NFATc1, CTSK, and TRAP were analyzed by qPCR using RNAs extracted from bone tissues from each experimental mouse group. N.s., not significant. Images presented are representative of $\geq 3$ sections for each group, and data are expressed as means $\pm S D$. ${ }^{*} P \leq 0.05,{ }^{\star *} P \leq 0.01$, ${ }^{\star * *} \mathrm{P} \leq 0.005$ when compared with vehicle control. 

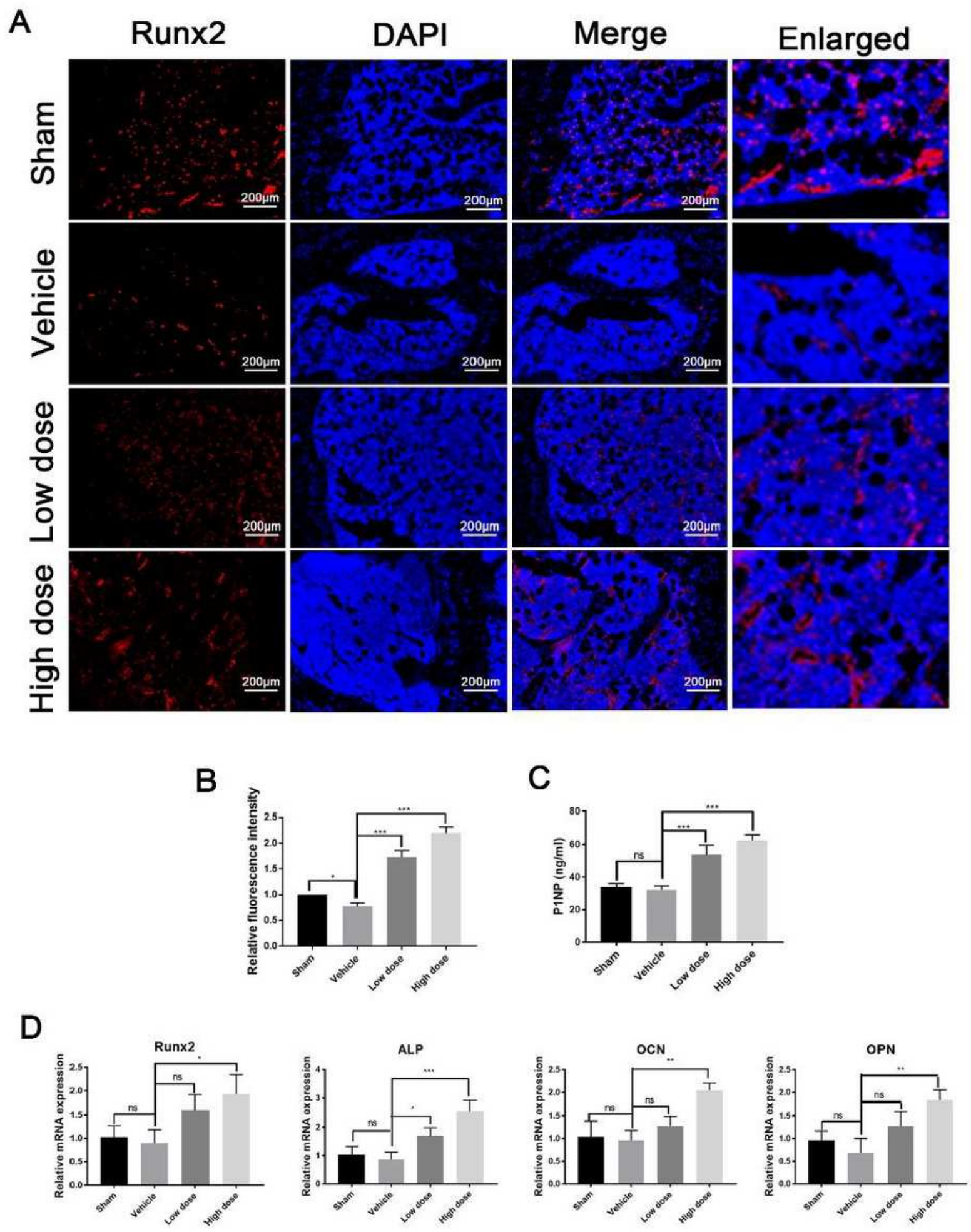

\section{Figure 7}

Administration of CSB6B promoted osteoblast activity in OVX-induced osteoporosis. A) Immunofluorescence analysis of the expression of Runx2 in tibial tissue sections from each experimental mouse group. Sections were counterstained with DAPI. Scale bars, $200 \mu \mathrm{m}$. B) The relative fluorescence intensity of Runx2 was quantified. C) To assess the relative levels of osteoblastic bone formation activity in each experimental mouse group, serum concentrations of P1NP $(n=6)$ were measured by ELISA. D) 
Osteoblast marker genes such as Runx2, ALP, OCN, and OPN were analyzed by qPCR using RNAs extracted from bone tissues from each experimental mouse group. N.s., not significant. Images presented are representative of $\geq 3$ sections for each group, and data are expressed as means $\pm S D$. ${ }^{*} P \leq 0.05$, ${ }^{\star} \mathrm{P} \leq 0.01,{ }^{\star * \star} \mathrm{P} \leq 0.005$ when compared with vehicle control. 